\title{
Exact Scalable Sensitivity Analysis for the Next Release Problem
}

\author{
MARK HARMAN and JENS KRINKE, University College London \\ INMACULADA MEDINA-BULO and FRANCISCO PALOMO-LOZANO, University of Cádiz \\ JIAN REN and SHIN YOO, University College London
}

\begin{abstract}
The nature of the requirements analysis problem, based as it is on uncertain and often inaccurate estimates of costs and effort, makes sensitivity analysis important. Sensitivity analysis allows the decision maker to identify those requirements and budgets that are particularly sensitive to misestimation. However, finding scalable sensitivity analysis techniques is not easy because the underlying optimization problem is NP-hard. This article introduces an approach to sensitivity analysis based on exact optimization. We implemented this approach as a tool, OATSAC, which allowed us to experimentally evaluate the scalability and applicability of Requirements Sensitivity Analysis (RSA). Our results show that OATSAC scales sufficiently well for practical applications in Requirements Sensitivity Analysis. We also show how the sensitivity analysis can yield insights into difficult and otherwise obscure interactions between budgets, requirements costs, and estimate inaccuracies using a real-world case study.
\end{abstract}

Categories and Subject Descriptors: D.2.1 [Software Engineering]: Requirements/Specifications-Tools

General Terms: Algorithms

Additional Key Words and Phrases: Next release problem, sensitivity analysis, requirement engineering

ACM Reference Format:

Mark Harman, Jens Krinke, Inmaculada Medina-Bulo, Francisco Palomo-Lozano, Jian Ren, and Shin Yoo. 2014. Exact scalable sensitivity analysis for the next release problem. ACM Trans. Softw. Eng. Methodol. 23, 2, Article 19 (March 2014), 31 pages.

DOI: http://dx.doi.org/10.1145/2537853

\section{INTRODUCTION}

Selecting a set of requirements for the next release of a software system is a complex and demanding problem. However, making good engineering and business judgments concerning these requirements is crucial because the decision process takes place so early in the development of the next release [Cheng and Atlee 2007]. The problem of choosing the optimal set of requirements to include in the next release of a software system has become known as the Next Release Problem (NRP) [Bagnall et al. 2001; Zhang et al. 2007].

The number of choices increases exponentially in the number of requirements, making this a nontrivial problem. The optimization problem that underlies it is NP-hard. The situation is further complicated by the uncertainties inherent to the decision making process. The choice of requirements for the next release is affected by the

This work was partially funded by the Spanish Ministry of Science and Innovation under the National Program for Research, Development and Innovation, project MoD-SOA (TIN2011-27242). It was also supported by the U.K. Engineering and Physical Sciences Research Council (grant numbers EP/D050863, EP/G060525). Data from the EPSRC-funded portions of this work may be available by contacting Dr. Yoo. Note that intellectual property or other restrictions may prevent the full disclosure of this data.

Corresponding author's email: shin.yoo@ucl.ac.uk.

Permission to make digital or hard copies of part or all of this work for personal or classroom use is granted without fee provided that copies are not made or distributed for profit or commercial advantage and that copies show this notice on the first page or initial screen of a display along with the full citation. Copyrights for components of this work owned by others than ACM must be honored. Abstracting with credit is permitted. To copy otherwise, to republish, to post on servers, to redistribute to lists, or to use any component of this work in other works requires prior specific permission and/or a fee. Request permissions from permissions@acm.org. (c) 2014 ACM 1049-331X/2014/03-ART19 $\$ 15.00$

DOI: http://dx.doi.org/10.1145/2537853 
expected cost and revenue that will accrue from the inclusion of each candidate requirement.

Unfortunately, a quantitative assessment of development cost and expected revenue can only be based on estimations at decision time. It is well known that such software engineering estimates can be inaccurate [Shepperd 2007]. Even if the requirements engineer is a perfect decision maker, their decisions might therefore be wrong because of the unavoidable inaccuracy of the estimates upon which they are based. An inaccuracy in the estimate of an attribute of one requirement may have a very different impact on the optimal choice of requirements compared to the same inaccuracy concerning the same attribute but of a different requirement. This raises a crucial question, upon which the entire requirements decision process rests.

Which estimates have the greatest impact upon the optimal requirements choice for the next release?

By identifying the budget values and requirements choices that have a higher impact on the decision, the decision maker can choose to allocate greater resources to the estimation process for these 'sensitive' inputs. This has the aim of improving the quality of the information available where it counts, making best use of available resources. We term the problem of ordering requirements' attributes according to their impact on the choice of requirements for the next release as the Requirements Sensitivity Analysis (RSA) problem.

Sensitivity applies to an individual attribute of a specific requirement for which small changes in attribute value have disproportionately large effects on the composition of the optimal requirements set. Sensitivity can also apply to an entire project budget for which the value of some attribute of many requirements has a disproportionate effect. We can also focus on the sensitivity of an attribute or budget at specific levels of inaccuracy. For instance, it can happen that a budget is most sensitive within a narrow range of potential estimate inaccuracy or that an attribute is only sensitive above a certain level of inaccuracy. Using sensitivity analysis, we can direct the decision maker to those areas of specific sensitivity and thereby help to inform the decision making process.

The relationship between cost, revenue, and inaccuracy cannot be easily understood without automated decision support, because small changes in the value of a certain attribute can have dramatic consequences on the overall solution. This is one of the reasons why the problem of determining the ideal requirements set is so hard. Our approach to the RSA problem is therefore to identify those attributes and budgets for which the sensitivity is unusually high for a given level of inaccuracy simply because of the particular happenstance of attribute value distribution. Recent work has indicated that Search-Based Optimization of the NRP produces human-competitive results, indicating that these approaches provide a good basis for decision support [de Souza et al. 2010].

In order to identify peculiarly sensitive values, our overall approach to RSA is to use multiple optimizations, each of which models a possible inaccuracy in requirements. In this manner, we follow the one-at-a-time (OAT) approach to sensitivity analysis, used in other areas of sensitive analysis [Saltelli et al. 2000]. However, to the best of our knowledge, we are the first authors to address this problem for requirements analysis.

We compute multiple NRP optimizations. On each occasion we perturb the value of a single attribute of a single requirement in order to mimic the effect of inaccurate estimation. We perturb by moving values both upwards and downwards, to capture the effect of underestimates as well as overestimates. We perform small step size perturbations of $5 \%$ from $-50 \%$ to $+50 \%$ of the unperturbed attribute value. Each execution of the NRP optimization algorithm therefore constructs a solution set of 
requirements for an instance of the problem in which one estimate was inaccurate by a given amount.

Much of the previous work on optimization algorithms for the NRP and release planning has concerned the application of metaheuristic search algorithms [Bagnall et al. 2001; Baker et al. 2006; Greer and Ruhe 2004]. Metaheuristic algorithms have been favored, partly because they scale well and handle multiple objectives [Saliu and Ruhe 2007a; Zhang et al. 2007]. By contrast, exact algorithms from the Operations Research literature that locate a globally optimal solution have been less widely used. Furthermore, though exact techniques have been previously applied to the NRP and release planning [van den Akker et al. 2005], their scalability has not been explored in the literature on NRP.

In order to attack the RSA problem using multiple executions of search-based optimization, we need to use an exact algorithm. If we use an inexact algorithm, we cannot be sure whether variations in results obtained from original and perturbed NRP problems accrue from inherent sensitivity or merely from the nature of the algorithms used to optimize. However, in choosing to use an exact approach to optimization, we are confronted with the issue of scalability.

In this article, we introduce and evaluate our approach to the RSA problem and a variant of the Nemhauser-Ullmann's algorithm [Nemhauser and Ullmann 1969] for exact optimization of each NRP instance. Since the algorithm must be run $A \times M$ times, where $A$ is the number of attributes to be perturbed and $M$ is the number of perturbations applied, we experimentally study and report on the scalability of the approach to demonstrate that it is practical. We also demonstrate that our approach is useful in practice, presenting three case studies of its use on a real-world RSA problem. The case studies reveal both peculiarly sensitive attributes and sensitive budgets.

The primary contributions of this article are as follows.

-We introduce the exact RSA problem and an OAT solution using the NemhauserUllmann's exact optimization algorithm as a solver for each OAT step.

-We implemented our approach in a tool, OATSAC (One At a Time Sensitivity Analysis for Cost-benefit) $)^{1}$, and used it to construct an experimental study of the scalability of the approach. The results of the experimental study indicate that the approach scales well for both size and complexity of NRP problems. The size is measured simply in terms of the number of requirements from which the NRP choice has to be made. The search-space size is exponential in this parameter. For complexity, we use the degree of correlation between cost and value, since high correlations usually denote complex (and therefore potentially less scalable) problem instances. The results of the experimental study provide evidence to support the claim that our approach is scalable and therefore practical.

-We report the results of a case study in which the approach was applied to the Motorola NRP requirements set (see Section 6.1 for details). The results show how a decision maker can use our approach to identify anomalous cases, both in terms of requirement attributes that are especially sensitive and also entire budgets for which the decision problem is sensitive. Identification of sensitive budgets allows the decision maker to negotiate for a more stable (less sensitive) budget, thereby addressing business concerns. Identification of attribute sensitivity allows the decision makers to target resources on sensitive attribute estimation, thereby addressing management and engineering concerns. The fact that we found cases of both kinds of sensitivity in our real-world case study provides evidence to support the claim that our approach may be useful in practice.

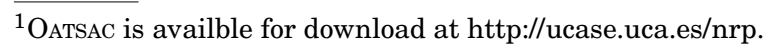


The organization of the rest of the article is as follows: Section 2 introduces the idea of Requirements Sensitivity Analysis, based on iterative solutions to perturbed NRP instances. Section 3 introduces the tool, OATSAC, which implements our approach and discuses the algorithmic choices involved in producing the exact and scalable results for NRP perturbations. Section 4 presents the research questions, the answers to which are presented in Sections 5 and 6. In Section 7, we extend our analysis to interactions between two requirements estimates (a problem we term the second order interaction problem), and in Section 8, we describe the actionable findings that a decision maker might exploit arising from our case study on the Motorola dataset. Section 9 considers the threats to validity and limitations of our work, while Section 10 presents related work on the NRP and sensitivity analysis. Section 11 discusses implications for subsequent work and Section 12 presents directions for future work. Finally, Section 13 concludes.

\section{REQUIREMENTS SENSITIVITY ANALYSIS}

Sensitivity analysis (SA) consists of assessing the contribution to the overall uncertainty of a solution that accrues from the individual uncertainty due to some specific element of the solution [Helton et al. 2006]. In this article, we adopt a one-at-a-time (OAT) approach to sensitivity analysis [Saltelli et al. 2000], which is also referred to as Local Sensitivity Analysis [Saltelli et al. 2008] and Nominal Range Sensitivity Analysis [Frey and Patil 2002] in the literature.

OAT methods vary one parameter at a time repeatedly, while all of the other parameters are maintained at their fixed, baseline values. OAT is the most popular SA practice in the literature [Saltelli and Annoni 2010]. Its strengths can be summarized as follows.

- The baseline vector provides a safe starting point from which to generate all perturbed versions. This minimizes the chance of generating invalid inputs.

-OAT guarantees that all impact is solely due to the perturbation in the input, provided that the model does not have a stochastic term.

-OAT does not produce type-I statistical errors; a nonzero impact always implies impacts from the perturbed input.

Hitherto, there has been no previous work on exact SA (OAT or any other form of SA) for the Next Release Problem in Requirements Engineering. This is an important omission in the previous literature, because the choice of requirements for the next release is a decision that is inherently and intrinsically based upon estimates that are widely believed to be unreliable. Nevertheless decisions concerning requirements do have to be taken and these decisions, coming as they do early in the lifecycle, can have a profound effect on the cost and effectiveness of the overall system. As this article shows, using OAT, it is possible to gain insight into those estimates that can have dramatic and otherwise unexpectedly high impact on the choice of requirements.

Our OAT approach is based on multiple iterations of the NRP optimization problem in search-based software engineering (SBSE), a subfield of software engineering that has grown rapidly in recent years [Colanzi et al. 2012; Freitas and Souza 2011; Harman 2007; Harman et al. 2012a, 2012b, 2012c; Zhang et al. 2008]. Each iteration caters for a different perturbed version of the original estimates. The NRP deals with the selection of a subset of requirements based on their desirability, for example, on their total expected revenue, while subject to constraints such as a limited budget [Bagnall et al. 2001]. The original formulation of NRP, due to Bagnall et al., considers an objective function whose value depends on the satisfaction of a set of customers via the inclusion of their demanded requirements in the next release of a complex software product; the aim of the problem being to select the subset of requirements (and, thus, of satisfied 
customers) maximizing the value of the aforementioned function without exceeding the company's budget.

More formally, let $S$ be a set containing $n$ requirements. For each requirement $x \in S$, let $\operatorname{cost}(x)$ represent its cost, and revenue $(x)$ its revenue. The extension of cost and revenue to $S$ can be simply defined as follows.

$$
\begin{aligned}
\operatorname{cost}(S) & =\sum_{x \in S} \operatorname{cost}(x), \\
\operatorname{revenue}(S) & =\sum_{x \in S} \operatorname{revenue}(x) .
\end{aligned}
$$

This NRP problem consists of selecting the subset of requirements $R$ with the highest revenue and with cost in budget. Let $B$ the budget, then $R \subseteq S$ is an optimal solution to the NRP problem if $\operatorname{cost}(R) \leq B$, and for all $Q \subseteq S$, the following property holds:

$$
\operatorname{cost}(Q) \leq B \rightarrow \operatorname{revenue}(Q) \leq \operatorname{revenue}(R) .
$$

We can achieve this by maximizing revenue $(R)$ subject to $R \subseteq S$ and $\operatorname{cost}(R) \leq B$. Let $c=\left[c_{1}, \ldots, c_{n}\right]$ and $r=\left[r_{1}, \ldots, r_{n}\right]$ the cost and revenue vectors for the $n$ requirements in $S$, and $s=\left[s_{1}, \ldots, s_{n}\right] \in\{0,1\}^{n}$ a solution vector, that is, a bitset identifying a subset of $S$. Then, we obtain the following equivalent optimization problem, which is a binary integer linear program.

$$
\begin{aligned}
\text { NRP : } & \max s \cdot r \\
& \text { subject to } \\
& s \cdot c \leq B \\
& s \in\{0,1\}^{n}
\end{aligned}
$$

It is clear from the preceding discussion that the NRP problem is essentially the classical 0/1 Knapsack Problem (KP). This is an NP-hard problem [Karp 1972]. This formulation assumes that costs and values are additive. If this assumption does not hold (e.g., there are synergies between requirements that reduce costs), then a more complex formulation is required. The exploration of such models and their impact on sensitivity analysis is an interesting topic for future work.

Previous work on solving the NRP problem has focused on metaheuristic optimization: while deterministic optimization techiques such as the greedy heuristic has been used [AlBourae et al. 2006], many have applied stochastic optimisation [Feather and Menzies 2002; Greer and Ruhe 2004; Jalali et al. 2008; Ngo-The and Ruhe 2009; Ruhe and Greer 2003; Ruhe and Ngo-The 2004; Zhang et al. 2008]. However, the inherent randomness in stochastic optimization poses new challenges to sensitivity analysis: if a what-if scenario yields a result different from the original instance of NRP, there is no way of knowing whether the difference is due to the stochastic nature of the approach or the impact of the parameter perturbation. Deterministic approximation such as greedy algorithms would eliminate the randomness but still at the cost of the optimality of the solution. Previous work has demonstrated that Greedy approaches are far from optimal in practice for requirements selection tasks [Ruhe et al. 2003].

To guarantee both the optimality of the solution and the lack of inexactness, it is necessary to use an exact algorithm for NRP: solutions obtained by exact algorithms will be optimal. However, this in turn raises the scalability concern, because OAT requires a large number of what-if problem solving experiments to produce a sufficiently detailed sensitivity analysis. Our research question addresses this question of scalability of the OAT approach combined with an exact algorithm. 


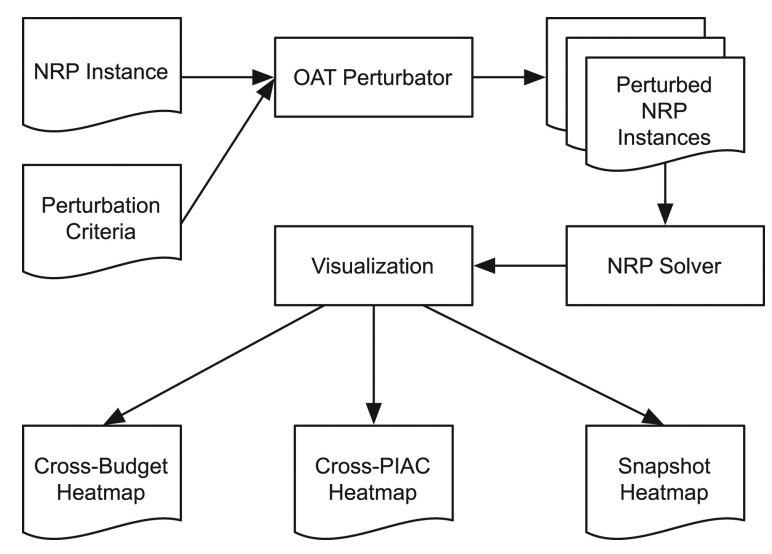

Fig. 1. Overall architecture of tool.

ALGORITHM 1: OAT Sensitivity Analysis Procedure

Input: An NRP instance, $P_{0}$, and a set of $n$ perturbation criteria, $I$

Output: Visualizations of $n$ different scenarios

(1) $\backslash$ Solve the original instance as a reference

(2) $S_{0} \leftarrow$ solve $\left(P_{0}\right)$

(3) $S \leftarrow \varnothing$

(4) Generate $n$ different perturbations

(5) $\quad P \leftarrow \varnothing$

(6) foreach $i \in I$

(7) $\quad P_{i} \leftarrow$ apply $i$ to $P_{0}$

(8) $\quad P \leftarrow P \bigcup\left\{P_{i}\right\}$

(9) Solve perturbed problem instances

(10) foreach $P_{i} \in P$

(11) $\quad S_{i} \leftarrow \operatorname{solve}\left(P_{i}\right)$

(12) $\quad S \leftarrow S \bigcup\left\{S_{i}\right\}$

(13) Visualize the results

(14) foreach $S_{i} \in S$

(15) Visualize the difference between $S_{i}$ and $S_{0}$

\section{THE OATSAC RSA TOOL}

Algorithm 1 outlines the overall procedure of one-at-a-time sensitivity analysis for NRP. Given the original NRP instance, $P_{0}$, and a set of predefined perturbations, we generate and solve $n$ different versions of the original instance. The results are then compared to the solution to the original instance for the visualization of the differences.

Figure 1 shows the overall architecture of our OAT sensitivity analysis tool, OATSAC (One At a Time Sensitivity Analysis for Cost-benefit), which consists of three main components: OAT perturbator, NRP solver, and visualization. OAT Perturbator accepts the original problem instance and a set of perturbation criteria as input, and generates a set of perturbed problem instances accordingly. These are fed into NRP Solver. While we use the Nemhauser-Ullmann's exact algorithm, any other NRP solver can be plugged in. The visualization component compares the different results from perturbed problem instances and highlights the impacts in three different types of heat-maps.

We use the term PIAC (percentage of increase in actual cost) to denote an estimate inaccuracy. The PIAC is the degree to which the estimator underestimated the true cost (since the actual cost is increased). A PIAC value of $x$ indicates the the actual cost 
is $x \%$ larger than the estimated cost. We allow both positive and negative values for PIAC, so that a negative value indicates an overestimate of cost.

-Snapshot Heatmap. These report the impact that a specific perturbation has on all requirements and budgets.

-Cross-PIAC Heatmap. These summarize the impact that each budget receives across the entire set of perturbations.

-Cross-Budget Heatmap. These summarize the impact of each perturbation across the entire budget range that the decision maker is interested in.

A crucial component of our tool is the choice of NRP solver to plug in. In this article, we report the results of the use of an implementation of the Nemhauser-Ullmann's algorithm, using this version of OATSAC to experimentally assess the scalability of this algorithm for solving multiple NRP problems as a component to RSA. We also replace this component with a faster, but less precise, greedy algorithm, showing how this is unsuited to the RSA problem, though it is faster, just because it is not exact.

\subsection{NRP Solver Module: Nemhauser-Ullmann}

The NRP Solver must repeatedly execute NRP instances, each of which has undergone some perturbation with respect to the original one to simulate estimation errors. Since there are many such executions required, and obtaining exact solutions is NP-hard, a great deal of care is required in the design and implementation of this module.

One important family of pseudopolynomial algorithms [Garey and Johnson 1978, 1979] that can be applied to the NRP can be obtained by dynamic programming. In particular, dynamic programming by costs is an efficient form of implementing the corresponding Bellman's equation when the number of requirements and the budget are moderate.

$$
z(S, B)= \begin{cases}0 & \text { if } S=\emptyset \\ z(S-\{x\}, B) & \text { if } S \neq \emptyset \wedge \operatorname{cost}(x)>B \\ \max \{z(S-\{x\}, B), z(S-\{x\}, B-\operatorname{cost}(x))+\operatorname{revenue}(x)\} & \text { otherwise. }\end{cases}
$$

In Eq. (2), the set $S$ represents a finite collection of requirements, $B$ is the budget, $z$ is the maximum revenue that can be achieved with any $R \subseteq S$ within the budget constraints, and $x$ is an arbitrary requirement in $S$ whose cost is $\operatorname{cost}(x)$ and its revenue is revenue $(x)$.

However, efficient implementations of this scheme typically require integer costs and thus they are not appropriate for RSA, because perturbations naturally produce fractional values. Of course, both the costs and the budget can be scaled to avoid fractional values while retaining precision, but then a previously modest size problem may be transformed into an unfeasibly demanding problem. Whether this unfeasibility occurs in practice will depend upon the specific instances considered. If there is a finegrained distinction between true costs, then scaling to ensure integer revenues will result in a (potentially) exponential explosion in problem size. Since a problem can have an arbitrary precision of revenues for requirements scores and costs (so arbitrarily fine grained revenues), we cannot be sure that this exponential increase will not occur in practice.

Therefore, though such a scaling approach might be applied in some instances, it is unlikely to provide a general solution in a reasonably large set of cases. The decision maker is unlikely to be an optimization expert and aware of the intricacies of the algorithm. Were such a non-specialist decision maker to rely on a technique that used scaling, we could never be sure that the algorithm at the heart of the approach would not (unexpectedly and inexplicably from the decision maker's point of view) fail to give 
an answer in reasonable time. For this reason we need to turn to an alternative way of providing exact scalable solutions to multiple instances of the NRP.

Approximation algorithms are far more efficient than exact algorithms, typically polynomial versus exponential. However, they might mislead the decision maker who is confronted by a sensitivity analysis involving hundreds of solutions corresponding to perturbations of a given NRP instance. Deviations from exact solutions may highlight the wrong requirements as being responsible of changes in the total revenue resulting in ill-informed decisions and sensitivity analysis that is simply wrong.

Therefore, we introduce Nemhauser-Ullmann's algorithm (NU) at the core of the NRP Solver component of OATSAC. We also implemented a greedy approach for comparison and present results (in Section 6.4) that demonstrate that its inaccuracies are problematic, as predicted, thereby motivating our proposed approach, based on an exact algorithm.

\section{ALGORITHM 2: Nemhauser-Ullmann algorithm for NRP}

Input: A set of $n$ requirements, $R$, and a budget, $B$

Output: A set of selected requirements, $S$

(1) Compute the optimal values

(2) $\quad m[0] \leftarrow z e r o()$

(3) for $k \leftarrow 1$ to $n$

(4) $\quad f \leftarrow \operatorname{translation}\left(m[k-1], \operatorname{cost}\left(R_{k}\right)\right.$, revenue $\left.\left(R_{k}\right)\right)$

(5) $\quad m[k] \leftarrow \operatorname{maximum}(m[k-1], f)$

(6) Recover an optimal solution

(7) $S \leftarrow \varnothing$

(8) for $k \leftarrow n$ to 1

(9) if $\operatorname{apply}(m[k], B) \neq \operatorname{apply}(m[k-1], B)$

$$
\begin{aligned}
S & \leftarrow S \cup\left\{R_{k}\right\} \\
B \leftarrow B-\operatorname{cost}\left(R_{k}\right) & \\
\text { return } & S
\end{aligned}
$$

Our particular rendition of NU for NRP is presented in Algorithm 2. The algorithm was implemented in standard $\mathrm{CH}$. Our description is based on the notion of staircase function. A staircase function is just an increasing function with a finite number of steps. These functions can be readily represented as lists of pairs containing the coordinates of each step.

First, the algorithm computes an array $m$ of $n+1$ staircase functions. The function $m[k]$ represents the optimal values that can be obtained by taking into account just the first $k$ requirements. ${ }^{2}$ When a new requirement is considered, the previous function is translated by adding the corresponding cost and revenue to each of its pairs. The resulting staircase function, $f$, may be better than the previous one at some points, while being worse at others. The maximum of both functions represents the combined optimal values.

Applying function $m[n]$ to cost $B$, which is the budget, we get the best revenue that can be obtained when all the requirements are taken into account. A subsequent simple backward search allows recovering the precise requirements involved in an optimal solution. A set, $S$, containing the indexes of those requirements is returned.

\footnotetext{
${ }^{2}$ Function $m[0]$ is just the zero function, a convenient special value.
} 
The technically challenging part of the algorithm is the efficient implementation of function maximum. This can be done in linear time with a method resembling the merge stage of mergesort. Observe that maximum is always invoked on two functions with the same number of points, say $p_{k-1}$ for $k \geq 1$ with $p_{0}=1$. Nevertheless, the resulting function can have up to $p_{k}=2 p_{k-1}$ points. Therefore, it follows that in the worst-case situation where such a pathological case would happen again and again, the algorithm would take an exponential amount of time in $n$.

\subsection{NRP Solver Module: Greedy Algorithm}

In order to investigate the benefits of using an exact algorithm, our case studies (Section 6) also report results for a greedy algorithm. Given a set of all requirements, $R$, and a budget, $B$, the greedy heuristic used in the study selects requirements in decreasing order of the revenue per cost ratio. When tied, requirements with higher revenue get prioritized; ties in revenue are, in turn, resolved in favor of the requirement with lower cost. The pseudocode of this greedy algorithm is presented as Algorithm 3.

\section{ALGORITHM 3: Outline of Greedy Approach for NRP}

Input: A set of $n$ requirements, $R$, and a budget, $B$

Output: A set of selected requirements, $S$

(3) Greedily select the most promising requirements in budget

(4) $\quad S \leftarrow \varnothing$

(5) $\quad k \leftarrow 1$

(6) while $k \leq n \wedge B \neq 0$

(7) $\quad$ if $\operatorname{cost}\left(R_{k}\right) \leq B$

(8) $\quad S \leftarrow\left\{R_{k}\right\} \cup S$

(9) $\quad B \leftarrow B-\operatorname{cost}\left(R_{k}\right)$

$$
k \leftarrow k+1
$$

\section{return $S$}

\section{RESEARCH QUESTIONS}

In order to evaluate whether our approach and the OATSAC tool that implements it are efficient and useful for the RSA problem, we conduct an experimental study concerning the efficiency of the approach and a set of case studies to explore its usefulness as a tool for revealing otherwise undiscovered sensitivity in budget and requirements. Finally, we compare the results obtained from the exact approach with those obtained from the faster, less precise approach, based on the greedy algorithm to explore the potential this might have to mislead the decision maker.

This is formalized as three research questions for which the rest of the article presents results. First, the need to use exact algorithms for sensitivity analysis raises an important question: what is the realistic cost of using an exact algorithm for sensitivity analysis of NRP? This question is, in turn, formulated more precisely as the following research questions.

RQ1. Impact Factors. Which factors affect the execution time of the exact algorithm for NRP? 
RQ2. Scalability Measurement. Based on RQ1, how well does the exact algorithm for NRP scale?

In addition to the experimental study of scalability, the article also presents the results of a case study that considers realistic scenarios in which our approach to sensitivity analysis can help the decision maker. The case study uses a real-world requirement dataset from Motorola and investigates the insights that can be provided to the decision maker that would not be possible without the aid of the sensitivity analysis.

RQ3. Insight. What are the possible benefits that a decision maker can expect from using the sensitivity analysis?

$R Q 1$ and $R Q 2$ are answered by statistically analyzing the measured execution time of the exact algorithm. $R Q 3$ naturally requires a more qualitative approach, based on usage scenarios for real-world NRP data.

\section{SCALABILITY STUDY}

The first two research questions are studied by generating synthetic problem instances of different sizes on which we apply the Nemhauser-Ullmann algorithm. The synthetic problem instances are not only varied on their sizes, but also on their difficulty, which will be explained in the following.

\subsection{Generating Problem Instances}

The research questions outlined in Section 4 require two control variables: the size of the problem (i.e., the number of requirements) and the difficulty of the problem instance. The scalability experiment uses synthetically generated problem instances that correspond to a predefined set of control variables.

While it is trivial to generate synthetic problem instances with predetermined problem sizes, generating problem instances with varying problem difficulties is not, in general. Fortunately, it is known that KP instances become harder when there is higher correlation between the cost and the revenue of items [Pisinger 2005]. Therefore, our synthetic instance generator controls the correlation factor between the cost and the revenue of the requirements in the problem instance in order to control its relative difficulty. Unlike Pisinger, we use Pearson's correlation as the measure of correlation and, thereby, this aspect of problem difficulty. We use Pearson's correlation because it is the most widely used and standard correlation measure used in statistical analysis, whereas Pisinger's correlation is more concerned with easing instance generation and theoretical analysis.

\subsection{Experimental Environment}

The scalability experiments were performed using a machine with an Intel Core i7 2.67 $\mathrm{GHz}$ CPU and 12GB RAM running Ubuntu 10.10. While the CPU provides a multicore environment, the algorithm used in the article has been implemented to run in a single thread. The execution time for each run of the algorithm has been measured using the standard Unix utility, time.

In order to answer $R Q 1$ and $R Q 2$, multiple instances of synthetic NRP instances were generated with varying numbers of requirements and relative problem difficulty levels, that is, the correlation between the cost and the revenue. In total, 15 different problem sizes ranging from 100 to 1500 requirements with steps of 100 were used; similarly, the correlation factor ranged from $0 \%$ (random costs and revenues) to $100 \%$ (equal cost and revenues) with steps of 5\%. This results in $15 \cdot 21=315$ problem configurations. In order to capture a sufficiently large sample of possible problem 
instances for each of the given control parameters, 50 different problem instances were generated from each of the 315 problem configurations. Each instance consists of a set of requirements with costs and revenues that are synthetically generated to uniformly sample the space of possible problem instances with respect to a fixed correlation. For each problem instance, the budget for NRP was set to the half of the total cost of all its requirements. In total, $315 \cdot 50=157,500$ different instances of NRP were solved using our implementation of the NU algorithm to provide results on scalability.

\subsection{Results and Analysis}

The results of our analysis of scalability are depicted in Figure 2. Figure 2 (upper graph) shows the box plots of the execution time of NU for NRP against problem instances with increasing number of requirements. Each datapoint corresponds to one execution of the algorithm on a synthetically generated problem instance. The box plots depict the distribution of 50 random problem instances generated for a single problem configuration.

The growing problem size increases the execution time of the algorithm almost polynomially, but the algorithm takes less than 1 minute for a problem instance with as many as 1,500 different requirements. In realistic scenarios, the number of requirements considered for a single release may be significantly fewer than 1,500, which makes our version of the Nemhauser-Ullmann algorithm scalable for multiple executions of NRP for use in an OAT approach to RSA.

Figure 2 (lower graph) shows the box plots of the execution time of NemhauserUllmann algorithm for NRP against problem instances with increasing correlation between the cost and the revenue of each requirement. As with the upper graph of Figure 2, each datapoint corresponds to a single execution of the algorithm. While the increasing correlation factor does result in increasing execution time, the impact is less than that of the increasing number of requirements and the growth is almost polynomial. The only exception to polynomial growth occurs when correlation is close to 1.0. However, the resulting execution time remains feasible, even in such extreme cases.

Table I presents the results for the average time data for configurations (i.e., a pair of correlation and problem size) with $10 \%$ correlation factor intervals. Given the number of requirements, $n$, and the correlation between the cost and the revenue of each requirement, $\rho(0 \leq \rho \leq 1)$, the following model of the time behavior, $t(n, \rho)$, fits the experimental data very well:

$$
t(n, \rho)=a n^{2} \exp \rho+b n^{2}+c n \log n .
$$

Coefficients for Eq. (3) are $a=6.56 \cdot 10^{-1}, b=4.67 \cdot 10^{-6}$, and $c=-1.14 \cdot 10^{-3}$. Figure 3 shows the plot of the experimental model of the time behavior together with its residuals. The $R^{2}$ value for the fit is 0.99 . We do not claim that the model explains the behavior of the algorithm under every circumstance, which would be clearly misleading considering that the worst-case execution time for NU is known to be $O\left(2^{n}\right)$ [Nemhauser and Ullmann 1969]. In pathological cases, our approach still may not apply. Nevertheless, as these experimental results indicate, there is strong evidence to indicate that our use of NU scales well to RSA.

\section{CASE STUDY}

The previous section provided evidence that our overall approach to RSA can scale to handle requirements problems with many requirements and with cost-revenue correlations that are known to cause exponential behavior in the underlying optimization algorithm. However, these results say nothing about whether the overall approach, 

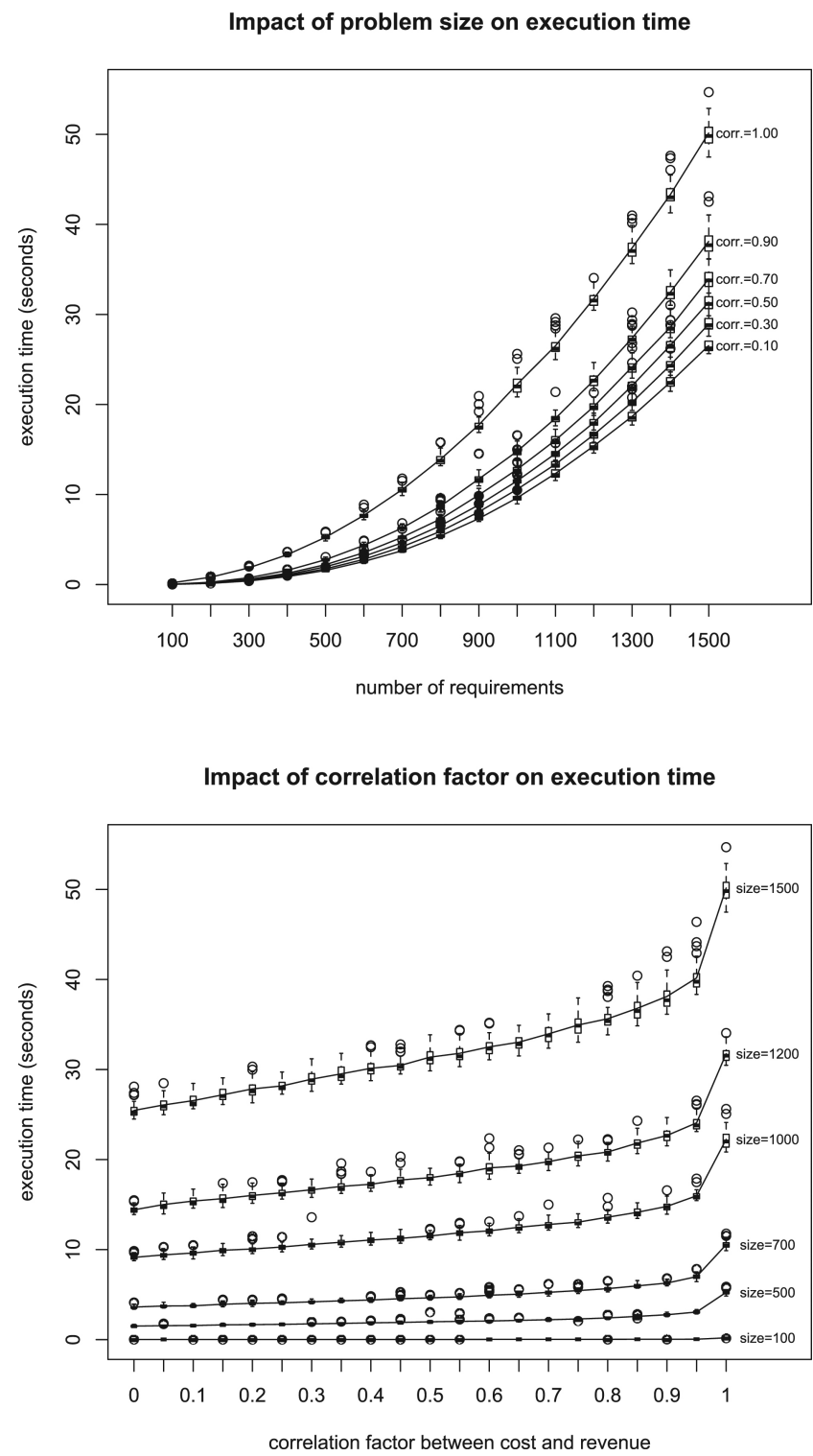

Fig. 2. The upper graph shows plots of execution time (seconds) for our implementation of the NemhauserUllmann algorithm against NRP instances with increasing number of requirements. The lower graph show plots of execution time (seconds) against NRP instances with increasing correlation between the cost and revenue of each requirement. It can be seen from the upper graph that execution time grows almost polynomially. From the lower graph, it can be seen that the impact of higher correlation values is also almost polynomial throughout the range of correlation values (except when the correlation value is very close to 1.0).

implemented in OATSAC, can help find interesting, important or insightful instances of sensitivity in real-world RSA problems.

To address this question, we present a detailed investigation of a requirements analysis problem from Motorola. In this problem, the requirements are potential features for mobile (cell) phones. While the specific details of the features and phones cannot be revealed for confidentiality reasons, this does not prevent us looking for interesting 
Table I. Execution Time (seconds) of Nemhauser-Ullmann's Algorithm for NRP for Different Problem Configurations

\begin{tabular}{rrrrrrrrrrrr}
\hline & \multicolumn{10}{c}{ Correlation } \\
\cline { 2 - 14 } & \multicolumn{1}{r|r}{$\%$} & $10 \%$ & $20 \%$ & $30 \%$ & $40 \%$ & $50 \%$ & $60 \%$ & $70 \%$ & $80 \%$ & $90 \%$ & $100 \%$ \\
\hline 100 & 0.02 & 0.02 & 0.02 & 0.02 & 0.02 & 0.02 & 0.02 & 0.03 & 0.03 & 0.04 & 0.20 \\
200 & 0.12 & 0.13 & 0.14 & 0.15 & 0.16 & 0.17 & 0.18 & 0.19 & 0.22 & 0.26 & 0.81 \\
300 & 0.38 & 0.40 & 0.42 & 0.45 & 0.47 & 0.50 & 0.53 & 0.58 & 0.65 & 0.76 & 1.86 \\
400 & 0.83 & 0.88 & 0.91 & 0.97 & 1.03 & 1.09 & 1.18 & 1.25 & 1.35 & 1.57 & 3.31 \\
500 & 1.50 & 1.57 & 1.67 & 1.75 & 1.86 & 1.98 & 2.07 & 2.21 & 2.41 & 2.76 & 5.25 \\
600 & 2.43 & 2.56 & 2.66 & 2.80 & 2.99 & 3.13 & 3.29 & 3.54 & 3.82 & 4.35 & 7.68 \\
700 & 3.62 & 3.76 & 4.03 & 4.20 & 4.40 & 4.64 & 4.93 & 5.26 & 5.66 & 6.28 & 10.55 \\
800 & 5.07 & 5.40 & 5.65 & 5.91 & 6.32 & 6.54 & 6.90 & 7.28 & 7.81 & 8.71 & 13.92 \\
900 & 6.99 & 7.33 & 7.70 & 8.07 & 8.46 & 8.84 & 9.31 & 9.95 & 10.56 & 11.73 & 17.73 \\
1000 & 9.13 & 9.62 & 10.07 & 10.58 & 11.06 & 11.51 & 12.08 & 12.79 & 13.60 & 14.80 & 22.25 \\
1100 & 11.63 & 12.33 & 12.88 & 13.36 & 14.02 & 14.55 & 15.53 & 16.04 & 16.94 & 18.46 & 26.51 \\
1200 & 14.42 & 15.39 & 16.02 & 16.64 & 17.26 & 17.98 & 19.07 & 19.76 & 20.84 & 22.67 & 31.75 \\
1300 & 17.82 & 18.67 & 19.68 & 20.25 & 21.21 & 22.08 & 23.07 & 24.17 & 25.57 & 27.37 & 37.39 \\
1400 & 21.48 & 22.48 & 23.49 & 24.37 & 25.46 & 26.57 & 27.58 & 28.58 & 30.32 & 32.48 & 43.32 \\
1500 & 25.46 & 26.55 & 27.85 & 28.93 & 30.14 & 31.38 & 32.51 & 33.96 & 35.64 & 38.12 & 50.16 \\
\hline
\end{tabular}
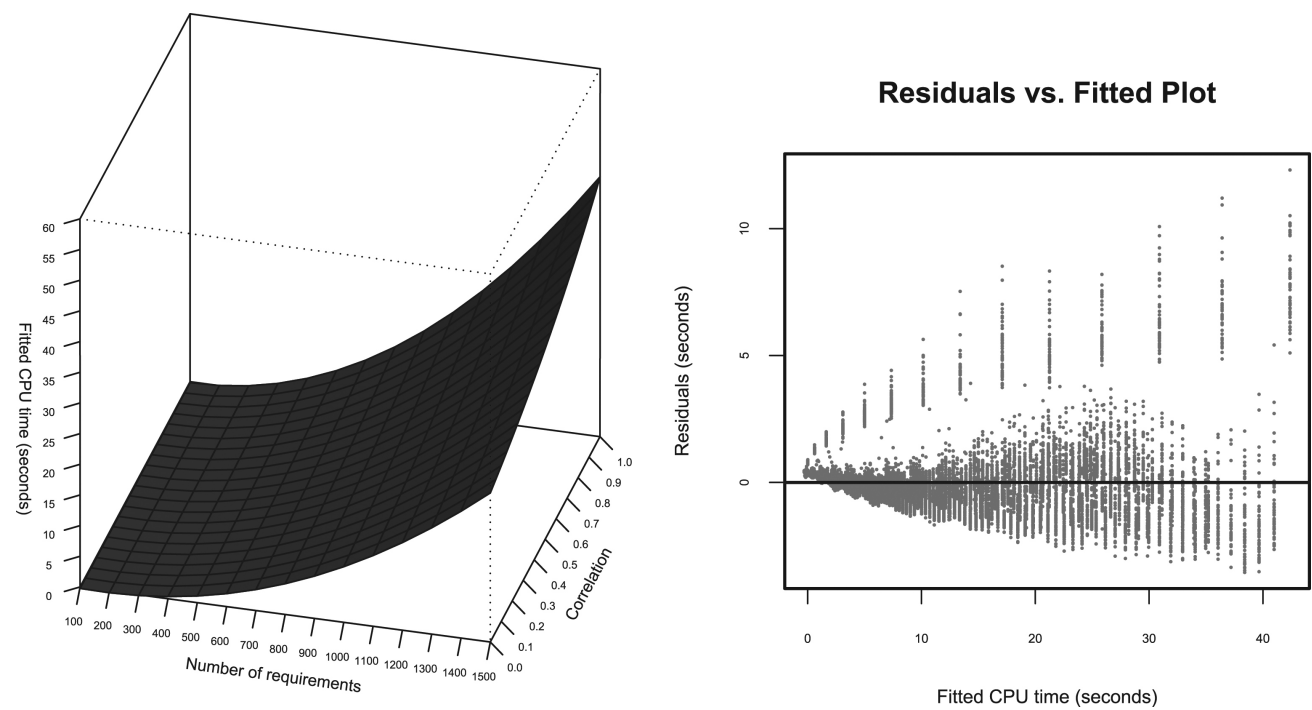

Fig. 3. The plot of the fit from the regression analysis, $t(n, \rho)=a n^{2} \exp \rho+b n^{2}+c n \log n$ and the plot of residuals. The $R^{2}$ of the fit is very high (0.99), indicating a very good fit to the observed experimental data.

and potentially insightful instances of peculiarly sensitive budgets and requirements and the interplay between them.

Using this dataset we do, indeed, find instances of both peculiarly sensitive requirements and peculiarly sensitive budget levels. Identifying and investigating these sensitivities would be very hard, if not impossible, without some form of decision support, such as that provided by our RSA approach, implemented in OATSAC.

\subsection{Requirements Data}

The case study considers a dataset from Motorola that contains 35 independent requirements. The dataset contains the cost of implementation and the expected revenue 
for each requirement, which form the cost function cost and the desirability function revenue in Section 2 , respectively. The expected revenue data have been provided by the customers, based on the desirability of having certain features in the next release. The dataset also contains 35 different budget values that are set by human experts.

\subsection{Sensitivity Analysis}

The sensitivity analysis we performed simulates misestimation of the cost of implementing a requirement by applying different PIAC values to the original cost of a requirement. For example, a positive PIAC value $p$ means the actual cost has increased by $p \%$ of the estimation (underestimation). Similarly, a negative PIAC value $-p$ means the actual cost has decreased by $p \%$ of the estimation (overestimation).

The sensitivity analysis uses PIAC values ranging from $-50 \%$ to $50 \%$ with 5 percentpoint intervals for each requirement. This is a matter of taste and can be varied without changing our approach. We find that this is a good balance of range and granularity. For $n$ requirements, this results in $21 n$ different 'what-if' scenarios that need to be solved for the sensitivity analysis, just for a single given budget proposal.

The total impact is computed separately for all positive PIAC values and for all negative PIAC values. The impact for each PIAC value is computed as the difference in overall revenue with respect to the solution of the unperturbed instance. The total impact of a misestimated requirement for each set of PIAC values is the sum of their impacts.

\subsection{Results and Insights}

In this section, we present the results of two case studies that illustrate how our approach can be used to support decision makers as they consider the various options available in negotiations over budgets. We consider two top-level scenarios for which sensitivity analysis can be useful in practice, applying both to the Motorola dataset.

In the first study, we consider a situation in which a budget level turns out to be particularly sensitive over all possible requirement inaccuracies. In the second study, we consider a scenario in which a particular requirement turns out to be atypically sensitive, regardless of the budget chosen. In this way, these two studies illustrate the two top-level concerns for the decision maker: sensitive budgets and sensitive requirements.

We will use the OATSAC heat map visualization of the sensitivity data in order to locate sensitive budgets and requirements. As the results show, the use of heat maps allows the decision maker to quickly and easily identify unusual areas of sensitivity within their candidate solution space. The scenarios show that sensitive budgets and requirements can easily be identified using the sensitivity heat map. However, they also reveal that the explanation for a particular sensitivity can be nontrivial and thereby non-obvious without the aid of OATSAC.

6.3.1. Sensitive Budgets. Consider the heat maps in Figure 4. These two heat maps show a perspective of our RSA results for the Motorola data set. The total impact of positive PIACs (Figure 4(a)) and negative PIACs (Figure 4(b)) on each budget (X-axis) for every requirement (Y-axis) is illustrated by the degree of darkness on the heat maps. Zero impact is represented as white color on both heat maps. Although darkness represents the difference on revenue caused by misestimation, in Figure 4(a), it represents the loss on revenue caused by +PIACs on cost of requirements, whilst in Figure 4(b), it represents the gain on revenue caused by -PIACs on cost of requirements. For example, consider requirement 13 for budget 450: the sum of all losses in revenue for the positive PIAC values is 6. 


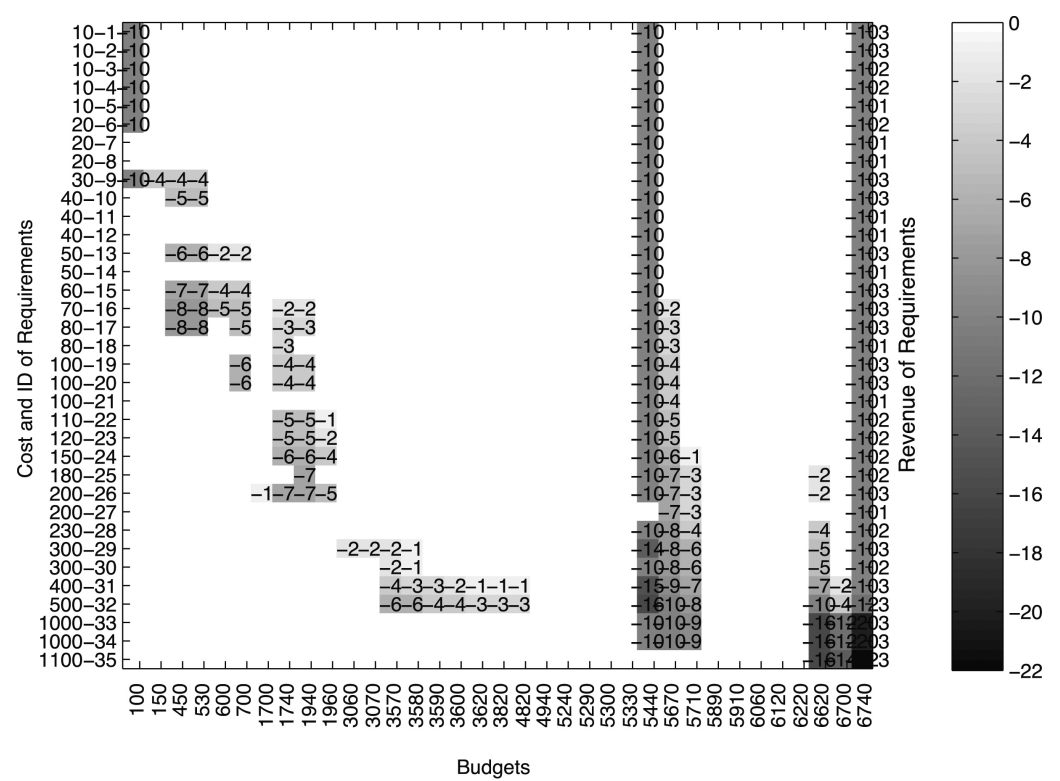

(a) Total impacts of +PIACs. Vertical bars indicate budgets $\{5440,6740\}$ are sensitive to +PIACs.
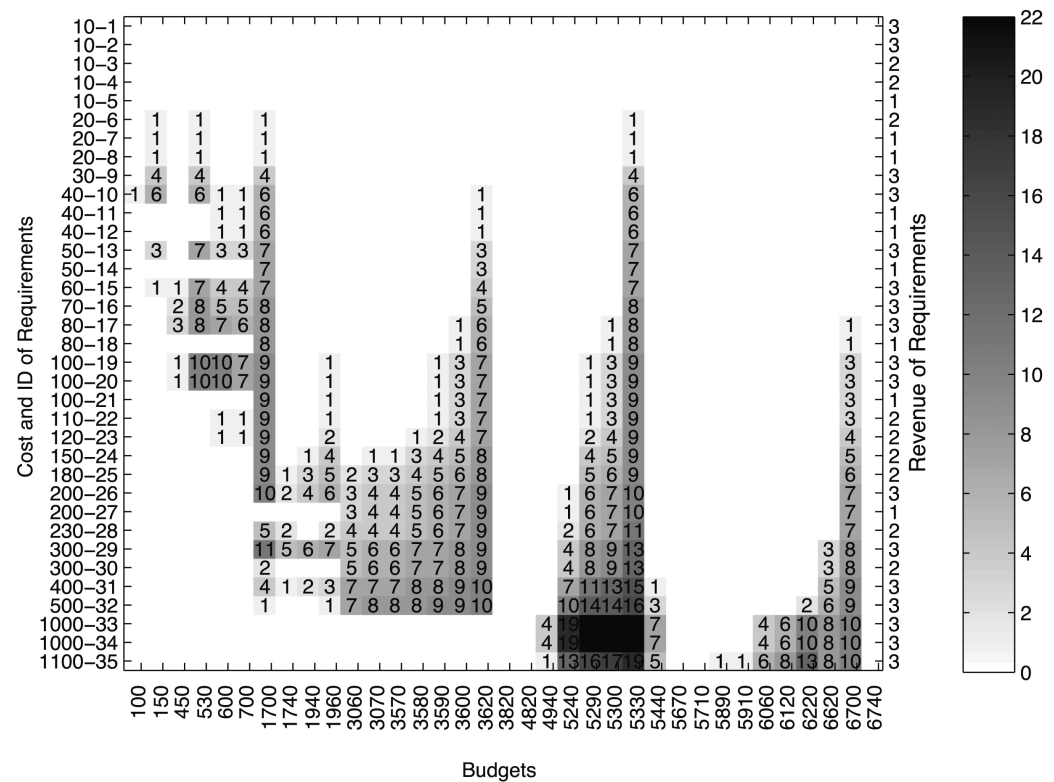

(b) Total impacts of -PIACs. Vertical bars indicate budgets $\{1700,3620,5330\}$ are sensitive to-PIACs.

Fig. 4. Budgets $\{5440,6740\}$ have zero surplus budget, which makes them sensitive to underestimates (+PIAC). On the other hand, budgets $\{1700,3620,5330\}$ have relatively more surplus budget $\{5.3 \%, 4.9 \%$, $13 \%\}$; as a result, they are sensitive to overestimates. 
Some allocations do not fully use the available budget creating a small budget surplus. This happens, for a solution $s$, when the total cost of solution $s$ is lower than the available budget, but no other solution exists that is within budget and enjoys a higher value. In such situations, there is a budget surplus (computed as the amount of budget available minus the total cost of the requirement selected for solution $s$ ). As shown in Figure 4, in general, budgets with surplus close to zero are more sensitive to +PIAC, and budgets with "rich" surplus are more sensitive to -PIACs. For example, the increasingly dark area on the left to the budget 5,330 in Figure 4(b) and the decreasingly dark area on the right to the budget 5,440 in Figure 4(a) are both caused by the increasing percentage of surplus budgets from left to right around that area.

For the heat map in Figure 4(a), starting from budget 5,440 to the two budgets on its right, because the surplus is growing away from the level of zero, it becomes easier to maintain the same level of overall revenue when the cost of the selected requirement was underestimated (+PIAC). This is reflected on the heat map as a trend of decreasing impact on the right of budget 5,440, meaning that budgets' ability of absorbing error is increasingly stronger when surplus is growing.

On the other hand, considering the four budgets on the left of budget 5,330 for the heat map in Figure 4(b), because the surplus is increasing to "richer" levels from left to the right, it becomes easier to accommodate the extra unselected requirements when selected requirement was overestimated (-PIAC). This is reflected on the heat map as the trend of increasing impact on the left of budget 5330, meaning that increasingly more revenue is added into solutions when surplus is growing.

More specifically, let us observe the dramatic sensitivity at the following budgets: $\{1,700,3,620,5,330,5,440,6,740\}$. Their sensitivity is revealed by the noticeably darker colors present around these budget levels. Closer analysis of the data reveals that these sensitive budgets can be classified into two types depending on the level of its surplus budget.

-Type 1. In budget 5,440 and 6,740, as shown in Figure 4(a), the original optimal requirement assignment has available very little surplus budget.

- Type 2. In budget 1,700, 3,620, and 5,330, as shown in Figure 4(b), there is a relatively large surplus available in budget: $\{5.3 \%, 4.9 \%, 13 \%\}$.

Clearly for Type 1 budgets, the lower the budget surplus, the less room for maneuver should there be for an inaccuracy in the estimation of costs. This can be expected to be a general trend. Indeed, for the Motorola data set, there is a good fit for an exponential function $f(x)=356.0 \exp (-139.8 x)$ to the graph of sensitivity to budget surplus, as shown in Figure 5.

One might be tempted to think this applies to all low surplus budgets, declaring these all to be sensitive budgets. However, this approach would be unreliable. For instance, observe that, though Figure 5 reveals a clear overall trend, it also contains notable outliers. This means that one cannot simply assume that low budget surplus leads to high sensitivity. For example, the budgets 100 and 600 have zero surplus, which is the tightest of all budgets for any solution. These turn out to be unremarkable and not particularly sensitive budgets. This is so because it turns out that there are plenty of unselected requirements with similar costs that can be substituted for misestimated requirements.

By contrast, the budget 5,330 is a rather sensitive one, as revealed by the heat map in Figure 4(b), yet it has a relatively high budget surplus. This is due to the fact that high budget surplus makes a budget sensitive to negative PIACs because of decreasing cost of selected requirement can make more room for either (1) accommodating extra unselected requirements, or (2) substitution by requirements combinations with higher revenue. This type of budget was classified as Type 2 sensitive budgets. 


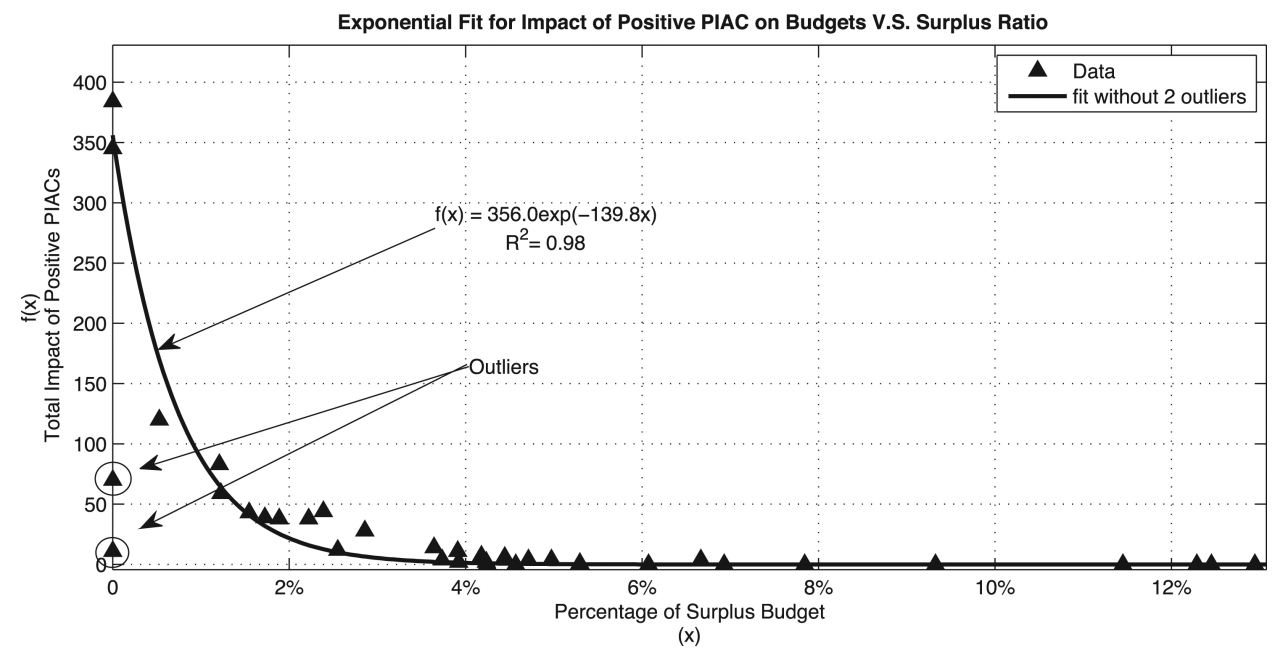

Fig. 5. Strong correlation between impacts of +PIAC and surplus percentage (Spearman Correlation Coefficient: $\rho=0.92$ ). However, two outliers: budgets 100 (lower) and 600, have zero surplus on budget but they are not sensitive to underestimated cost of requirement (+PIACs) because of the flexibility of substitutions.

Furthermore, one cannot assume, even for a budget identified as sensitive, that all requirements will be sensitive, nor that all levels of inaccuracy will be equally important. For example, consider again, the budget level 5,440, which was revealed to be a sensitive one for Figure 4(a). Observe that requirement 27 is not at all sensitive at this budget level, even though all other requirements are for this budget level. In this case, further detailed analysis reveals that this requirement has the same cost as another requirement, and that this means that the other requirements can be chosen in preference to it, making it unimportant at that particular budget level.

Once again, one might be tempted to adopt the assumption that all such "equal cost" requirements would be similarly insensitive, but once again, this would be a misplaced assumption; requirement 13 has identical cost to requirement 14, yet both are sensitive at this highly sensitive budget level of 5,440, as the heat map shows on Figure 4(a). This visual illustration of sensitivity can be useful to the decision maker. We discuss, in more detail, how a decision maker might find actionable results using our heat maps in Section 8.

6.3.2. Sensitive Requirements. The previous section illustrated how the OATSAC heat map can help to identify sensitive budget levels. In this section, we turn our attention to using OATSAC to identify sensitive requirements.

Consider the OATSAC heat map in Figure 6 which shows the impact of each requirement by each level of PIAC. The level of darkness on the heat map represents the degree of difference caused by corresponding PIAC (horizontal axis) on a specific requirement (vertical axis) summarized over all budgets. The corresponding requirements on the vertical axis are arranged in ascending ordered of their cost, and 'equal cost' requirements are arranged in descending order of their revenue.

In general, requirements with higher costs have higher sensitivity to errors, because applying the same percentage of error (PIAC) on the cost of requirements, the most expensive requirements tend to entail a larger absolute error. This can be expected to be a general trend. Indeed, as shown in Figure 7, the level of sensitivity of a requirement has a strong correlation with its cost. The statistical analysis also confirms this claim with a value of $\rho=0.94$ for the Spearman Correlation Coefficient. Requirement 


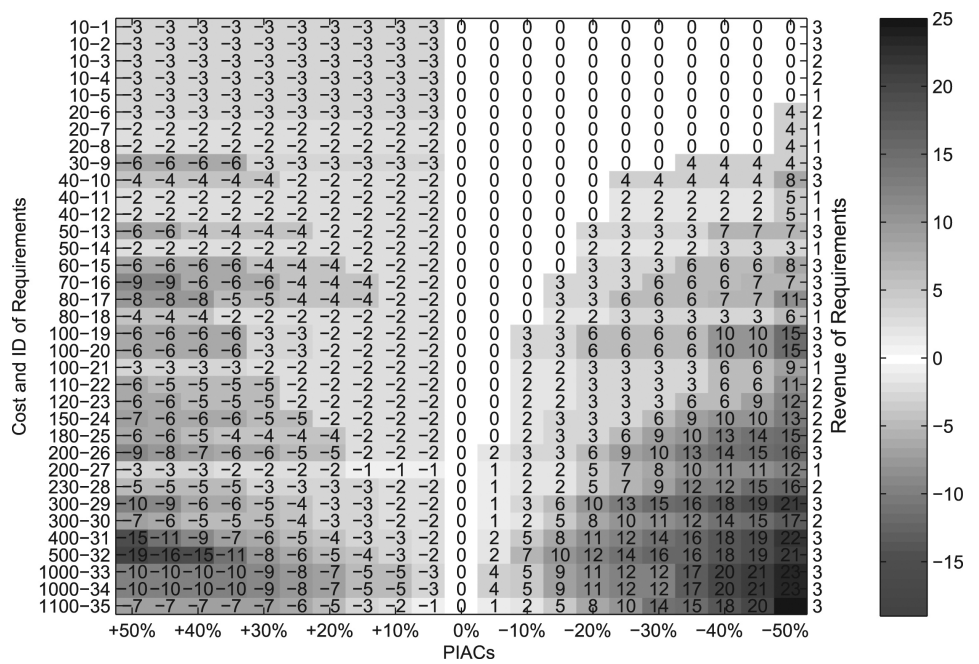

Fig. 6. The impact of each requirement with different levels of misestimation.

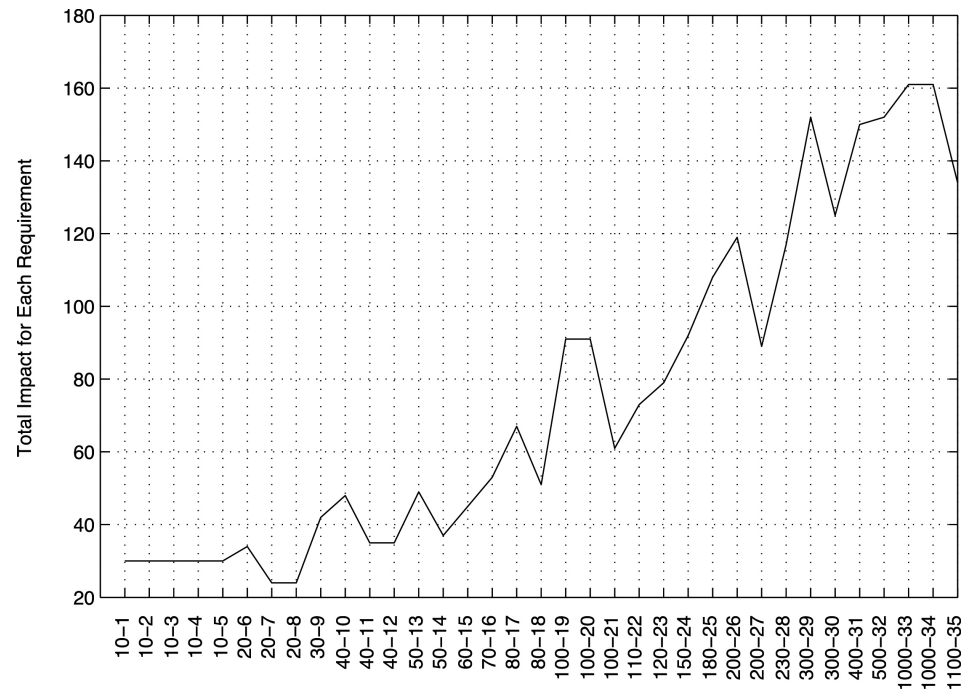

Requirement: Cost and ID

Fig. 7. Cost of requirements vs. total impact of each requirement. More expensive requirements (on the right) do not necessarily have more impact than the cheaper (i.e., to the left) ones. When the costs are the same, those with lower revenue (on the right) have lower impact, for example, these requirement sets have identical cost: $\{6,7,8\},\{10,11,12\},\{13,14\},\{17,18\},\{19,20,21\},\{26,27\}$, and $\{29,30\}$.

revenues also play an important role during the selection process. Naturally, one would expect that when two requirements have the same cost but different revenue, the requirement with lower revenue is always less sensitive.

However, though these are general expectations, there are exceptions, and the OATSAC heat map helps to reveal these exceptions. Requirement 35 has a higher cost and with the same revenue as requirement 34 , but, as revealed by the heat map in Figure 6 , requirement 35 has a relatively smaller impact than requirement 34 . 


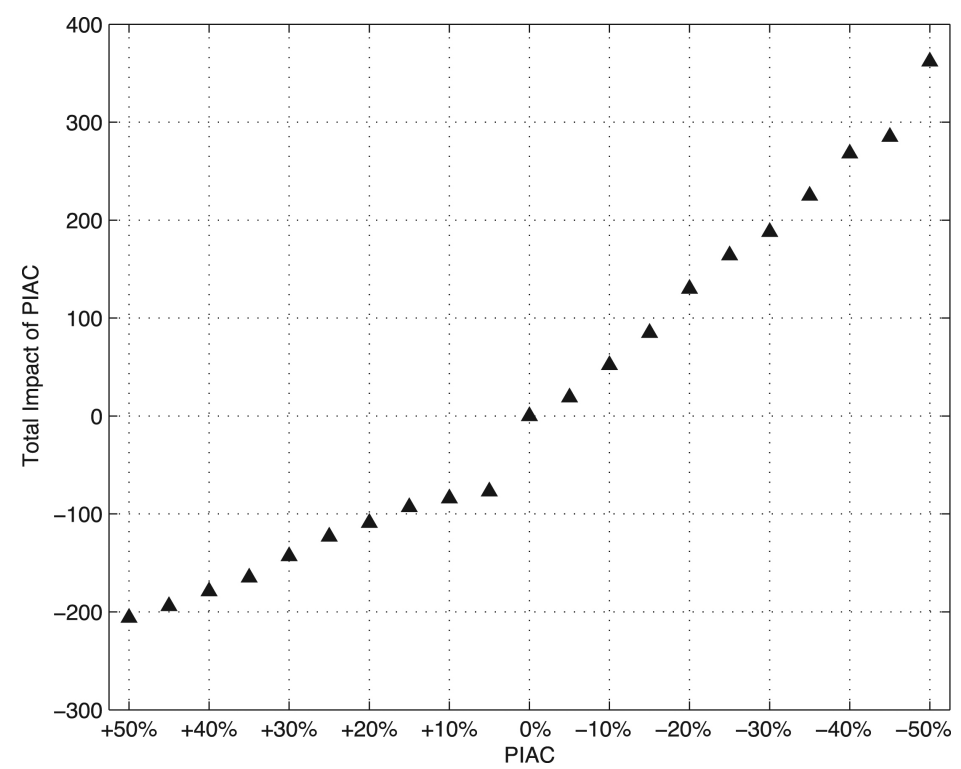

Fig. 8. Rank correlation between the total impact of each PIAC and PIAC value. The trend is monotonic.

6.3.3. Sensitivity to Specific Levels of Misestimation (PIAC). There is another interesting observation, as shown in Figure 8: underestimations on the cost of requirements (negative PIAC, shown on the right half of the figure) have higher absolute impact than overestimations. Closer examination of the data reveals that this is due to the large number of available substitute requirements in the Motorola dataset. That is, it turns out that often there are two or more equally good solutions with same revenue in this particular dataset so that the misestimation of one requirement value might not affect the overall solution; the solution can be retained by a like-for-like substitution. By contrast, when the PIAC is negative, indicating an underestimation of requirement cost, either more surplus budget becomes available, or the cost of some unselected requirement becomes sufficiently small that it can be accommodated within the increased surplus.

\subsection{Why the Use of an Exact Algorithm is Essential for RSA}

The greedy algorithm provides a nonstochastic approximation for NRP. Although the deterministic nature of the algorithm makes it an ideal candidate for an OAT approach, the errors in approximated solutions can mislead the decision maker. In this article, we demonstrate the negative impact of using a non-exact heuristic for SA. The greedy algorithm used in this comparison selects requirements in the descending order of their revenue-to-cost ratio, subject to the constraint that the total cost does not exceed the given budget.

Figure 9 illustrates the comparisons of the results for NU-OATSAC with GreedyOATSAC for a PIAC $=-50 \%$. While Greedy-OATSAC produces results that are superficially similar to NU-OATSAC, there are pairs of requirements and budgets that show very different levels of impact. Certain impacts are wrongly either ignored or exaggerated by Greedy-OATSAC. For example, the darker areas in the Greedy-OATSAC results represent lost revenues, which would be regarded as a significant impact. However, the NUOATSAC shows that the revenue can be retained. This illustrates the importance of using more expensive (but exact) algorithms for RSA. 


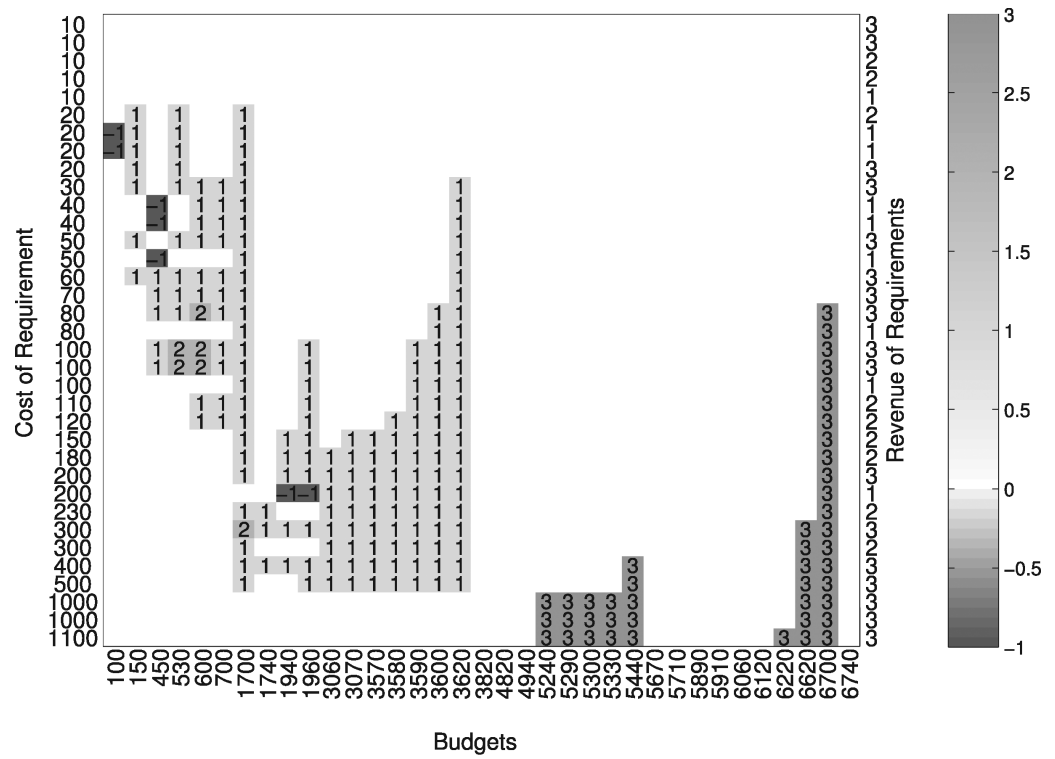

(a) Greedy algorithm.

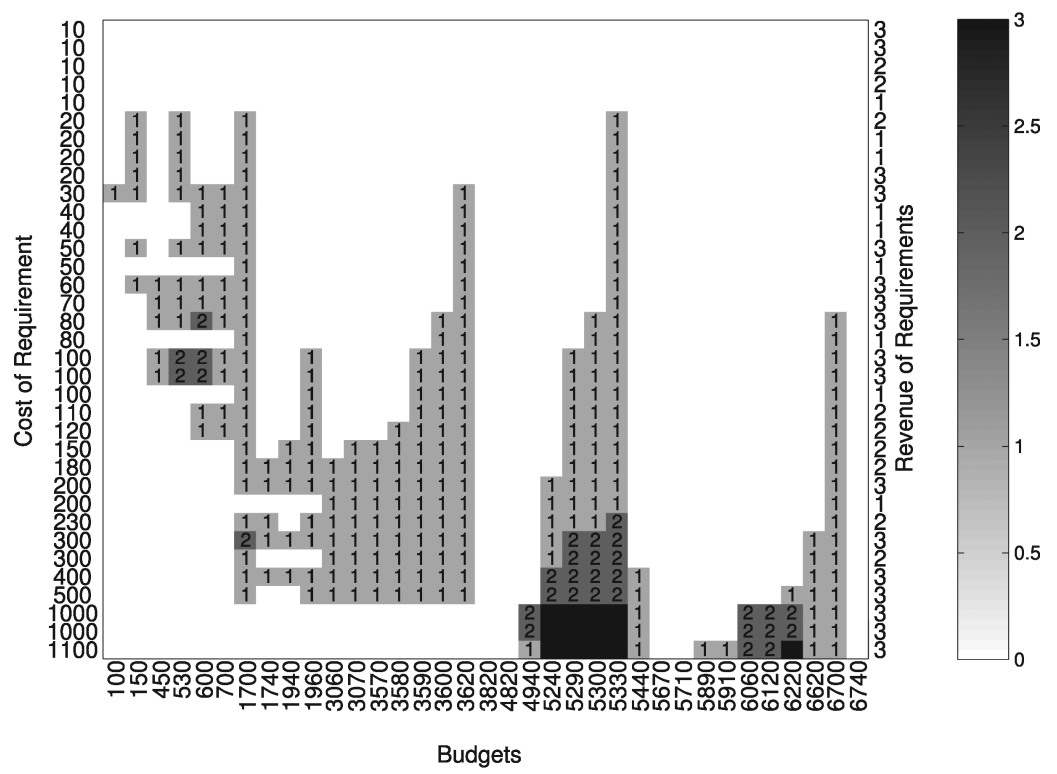

(b) Nemhauser-Ullmann's algorithm.

Fig. 9. Comparison of greedy and NU NRP Solver results. The two graphs show the impact on total revenue with $50 \%$ overestimation (PIAC $=-50 \%$ ) on the cost of each requirement according to each algorithm. Note that the greedy algorithm misreports many heat map values. This can mislead the decision marker. The NU algorithm is exact and so all reported heat map values are guaranteed to reflect only the impact of sensitivity on the NRP, while for greedy, the heat map results are confounded by the inherent imprecision of the algorithm. 


\section{CATERING FOR HIGHER ORDER ESTIMATE INACCURACY EFFECTS}

In this section, we consider the problem of interactions between estimate inaccuracies. Clearly, as the number of interactions between estimate inaccuracies increases, the computational cost of accounting for them grows exponentially. We call the problem of analyzing interactions between estimate inaccuracies involving two different requirements the second-order interaction problem. More generally, we use the term higher-order interaction for any interaction between inaccuracies involving $n$ requirements for $n>1$.

In order to cater for second-order interaction problem, we need a different approach to visualization. However, at this order, it remains possible to consider all possible interactions, giving the decision maker a complete picture of the possible impact of estimate inaccuracies, in which two inaccuracies occur simultaneously. The problem of analysis of higher orders remains a topic for future work (discussed briefly in Section 12).

We use a four-dimensional format to display the second-order interactions for a given level of estimate inaccuracy (PIAC). We use a three-dimensional plot for the axes relating to the two requirements to which the PIAC is applied and show the corresponding budget as the third axis. In order to capture the impact of the interaction between the two requirements for a given budget and PIAC level, we show this as a color intensity (the 'fourth' dimension). Naturally, this style of visualization is best viewed and explored interactively in color, though it can also be appreciated in black and white, through the corresponding shading.

In order to assess the impact of second-order effects, we measure the additional impact obtained from the interaction of the two requirements over and above the impact observed for the sum of each of the individual impacts. This provides a visualization of the additional effects due the interaction of estimate inaccuracies (the second-order effects). Note that the additional impact can be positive or negative, as it is the difference between the joint impact of two requirements and the sum of their individual impacts, and the former may be larger or smaller than the latter.

Figure 10 shows the effects of underestimates, when the degree of underestimation is the maximum considered in this article. That is, in this figure, the true cost is $50 \%$ greater than estimated, so PIAC $=+0.50)$. Figure 11 shows the effects of overestimates, when the degree of underestimation is the maximum considered in this article.

As can be seen from these two figures, the additional impacts that accrue from second-order effects tend to impact most on the more expensive budgets (the budget levels are ordered in increasing size of the vertical axis). The two figures also reveal that there are certain budget levels that suffer more than others from sensitivity to second-order estimate inaccuracies. Looking at these budgets we observed that there was a great deal of similarity between those budgets that are sensitive at the first order and those that are sensitive at the second order.

This may provide tentative evidence that the additional effects that accrue from higher-order effects are closely coupled to their first-order counterparts; budgets that are sensitive at the first-order level tend to be sensitive at higher orders. Of course, more examples need to be considered, and more analysis of higher order effects would be required to provide sufficient empirical evidence to support any generalization of this claim. It may also be possible to demonstrate a theoretical relationship between first-order effects and additional impacts at higher orders. However, this remains a problem for future work.

The effects of second-order interactions can also be seen for lower estimate inaccuracy levels (smaller absolute values of PIAC). These are shown in Figures 12 and 13 which show, respectively, additional second-order effects of under- and overestimation for four other PIAC levels. Naturally, the lower the estimate inaccuracy, the lower the impact of any and all estimate inaccuracies. However, we see the same overall pattern in these 


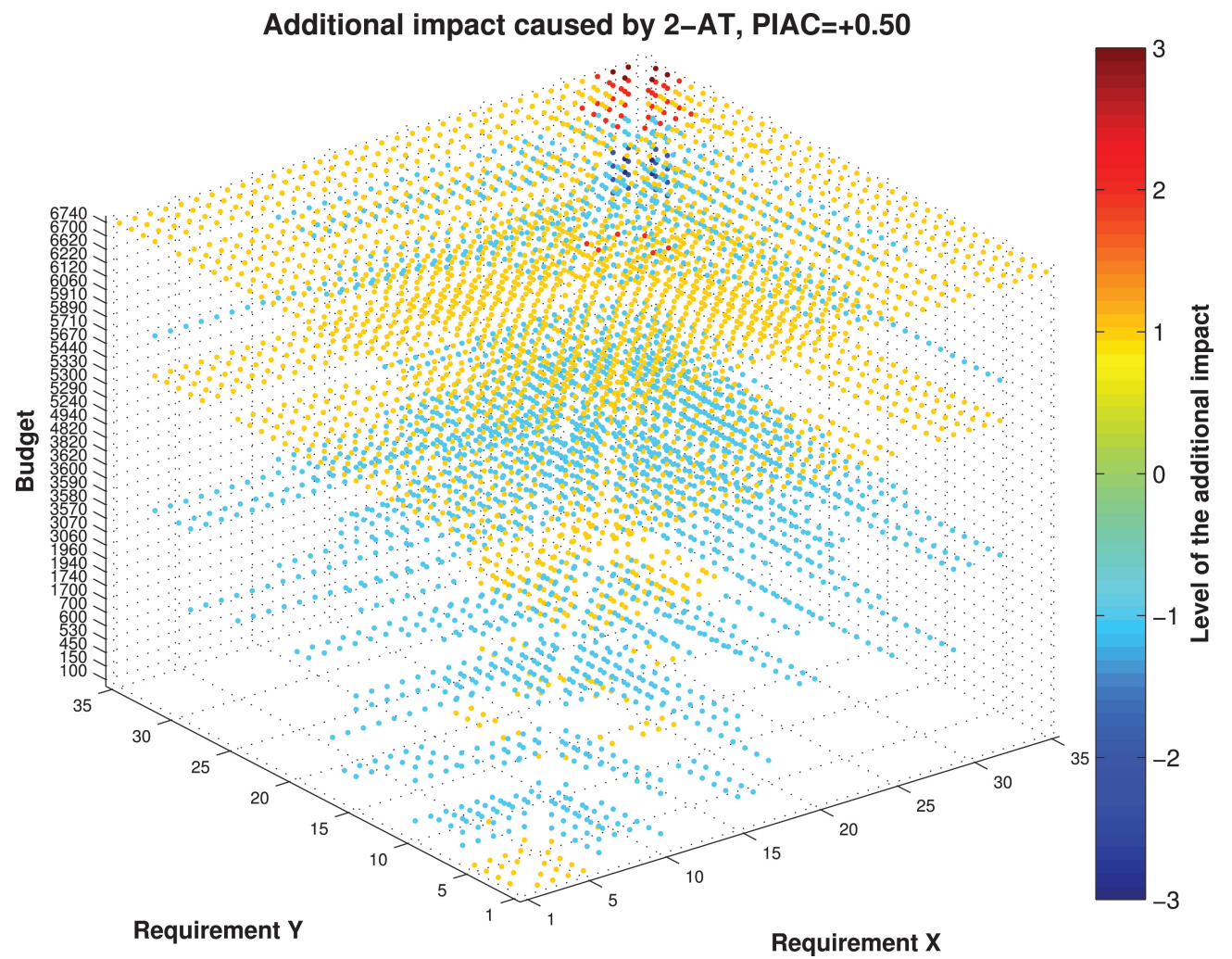

Fig. 10. Additional impact of second-order effects for $50 \%$ underestimated cost $(\mathrm{PIAC}=+0.50)$.

figures: certain budgets are more sensitive than others, and there is a general tendency for more expensive budgets to suffer from greater additional impact.

\section{HOW THE RESULTS CAN BE USED BY A SOFTWARE ENGINEER}

We saw (Figure 4) that there is a general principle empirically observed in the Motorola dataset that budgets with surplus close to zero are more sensitive to underestimation, while budgets with 'rich' surplus are more sensitive to overestimates. However, we also saw that there are sensitivities that can only be revealed by the analysis and do not follow this general principle. For example, the budget values 100 and 600 have zero surplus (tightest of all budgets) but are not particularly sensitive, while budget 5,330 is highly sensitive considering it has a relatively high budget surplus.

The decision maker can thus use the heat maps as a way to identify those budgets that are sensitive to estimate inaccuracies. This can feed into the negotiations the decision maker might have with other stake holders. Without the heat maps, the decision maker would simply have to assume that tight budgets would be sensitive and that high surplus provided a cushion of security. As we have seen, this would not be the best policy. There may be more room to maneuver in a tight budget scenario and any sense of security arising from a budget surplus might be a very false sense of security. The decision maker would thus be well advised to use the analysis afforded by the heat maps to back up their innate common sense and intuition.

Similarly, we observed that the degree of sensitivity of requirements varies (even within budget levels that are, themselves, found to be very sensitive). For example, the 


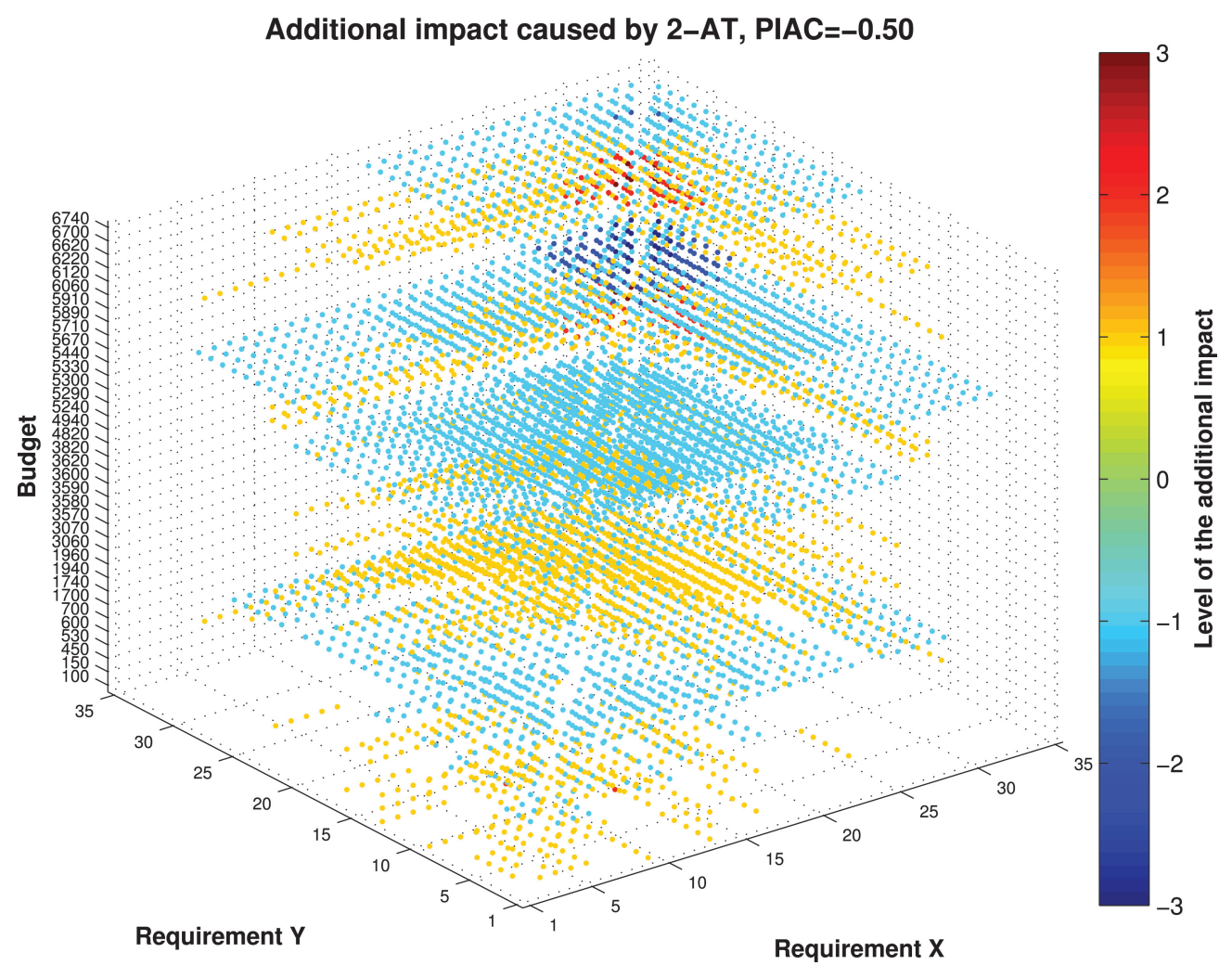

Fig. 11. Additional impact of second-order effects for $50 \%$ overestimated cost (PIAC $=-0.50)$.

budget 5,440 illustrates this point very clearly. Even for this highly sensitive budget level, there is one completely insensitive requirement that remains entirely unaffected by estimate inaccuracy.

These kinds of observations, taken directly from the heat maps, also provide information that can be useful to the decision maker. The analysis of the heatmaps reveals that it would be wise to seek to negotiate for a different budget if presented with a management case for an overall budget of 5,440. Furthermore, it supports the decision maker by providing him or her with a business case to underpin their negotiations. The decision maker might appeal: "this particular budget is simply a too risky budget and a dramatic reduction in risk can be achieved with a small budget modification."

Of course, such negotiation may prove to be either impossible or unsuccessful. However, even in such an unfortunate situation, our sensitivity analysis approach retains its usefulness, because the decision maker is both alerted to the need for particularly careful cost estimation and also to those requirements that require particular attention.

We have seen that the decision maker can identify highly sensitive budget levels and requirements in the Motorola dataset, providing some evidence that the use of our heat maps can provide actionable results to the software engineering decision maker. We cannot claim that all requirements problems will yield interesting actionable results, but even where they do not, this means that the manager will have additional confidence that 'there is nothing out of the ordinary' in the sensitivity of the budgets and requirements. 

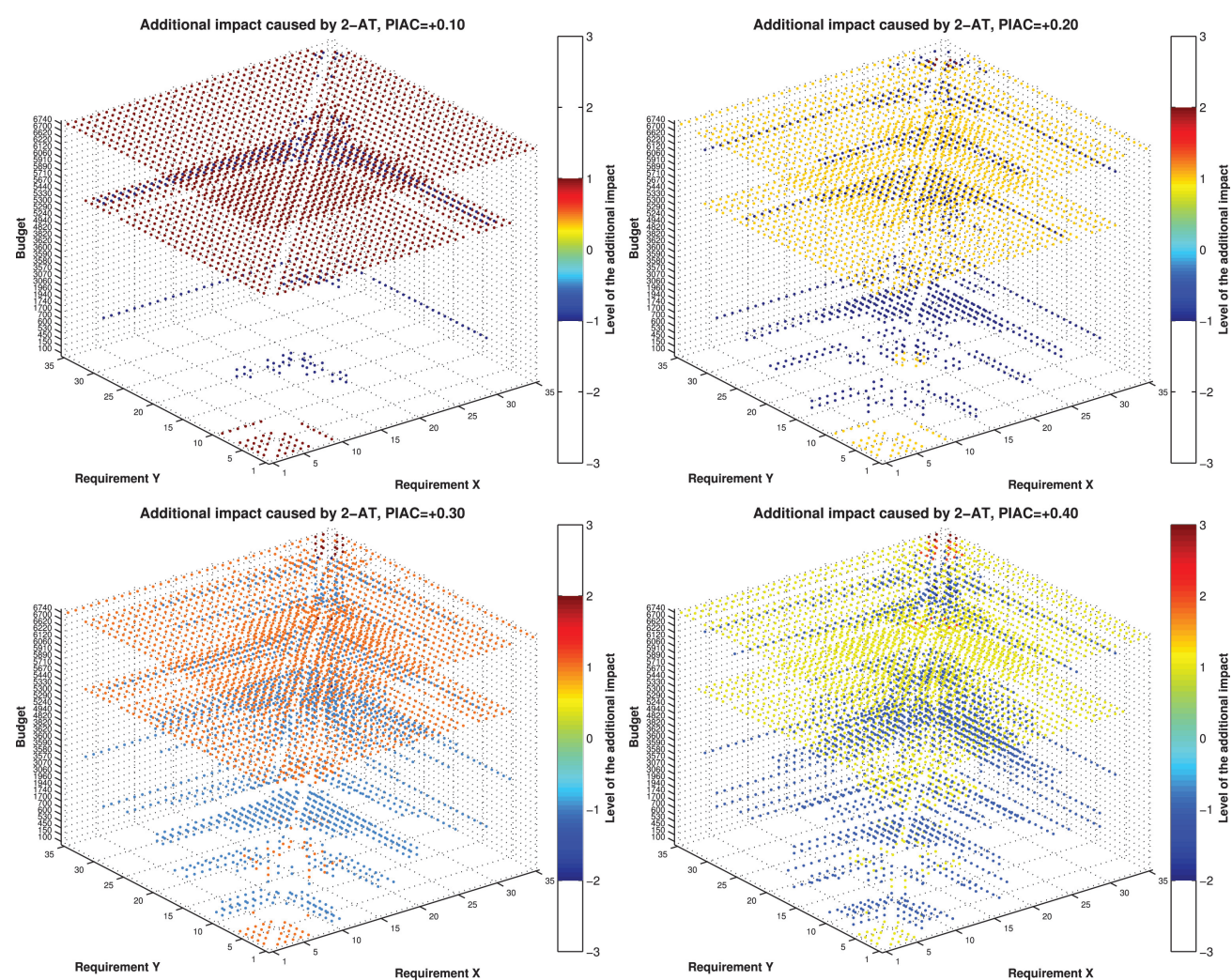

Fig. 12. Additional impact of second-order effects for positive PIAC (underestimated cost).

For the Motorola dataset, we have also seen that the reasons for sensitivity are far from obvious without the OATSAC sensitivity heat map. The subtlety of sensitivity is a motivation for the automated decision support approach advocated in this article. The Motorola dataset is a modest requirements problem, with only 35 different requirement choices. Though we make no claims about results that may be obtained for other requirement problems, it was clear that there were several subtleties revealed by our analysis, even in this relatively modest requirements set. We can therefore have measured confidence that there will be other interesting sensitivities elsewhere too and that these may lead to similarly actionable findings.

\section{THREATS TO VALIDITY}

Threats to internal validity concerns the factors that could have affected the observations made during the experimental evaluations. Any experimental evaluation of scalability is not free from environmental issues. The execution time was measured using Unix time utility. In order to control factors that could perturb our measures, the experiment was performed only after we ensured that pad peaks and other memory intensive tasks could be avoided as much as possible. We have repeated the experiment up to three times depending on the existence of outliers. Threats to external validity concerns the factors that prevent the generalization of the results. The scalability observed in the experimental evaluation clearly only applies to the specific choice of the problem definition and the algorithm. The same level of scalability may not be easily achievable for different classes of problems or exact algorithms. However, the NRP 

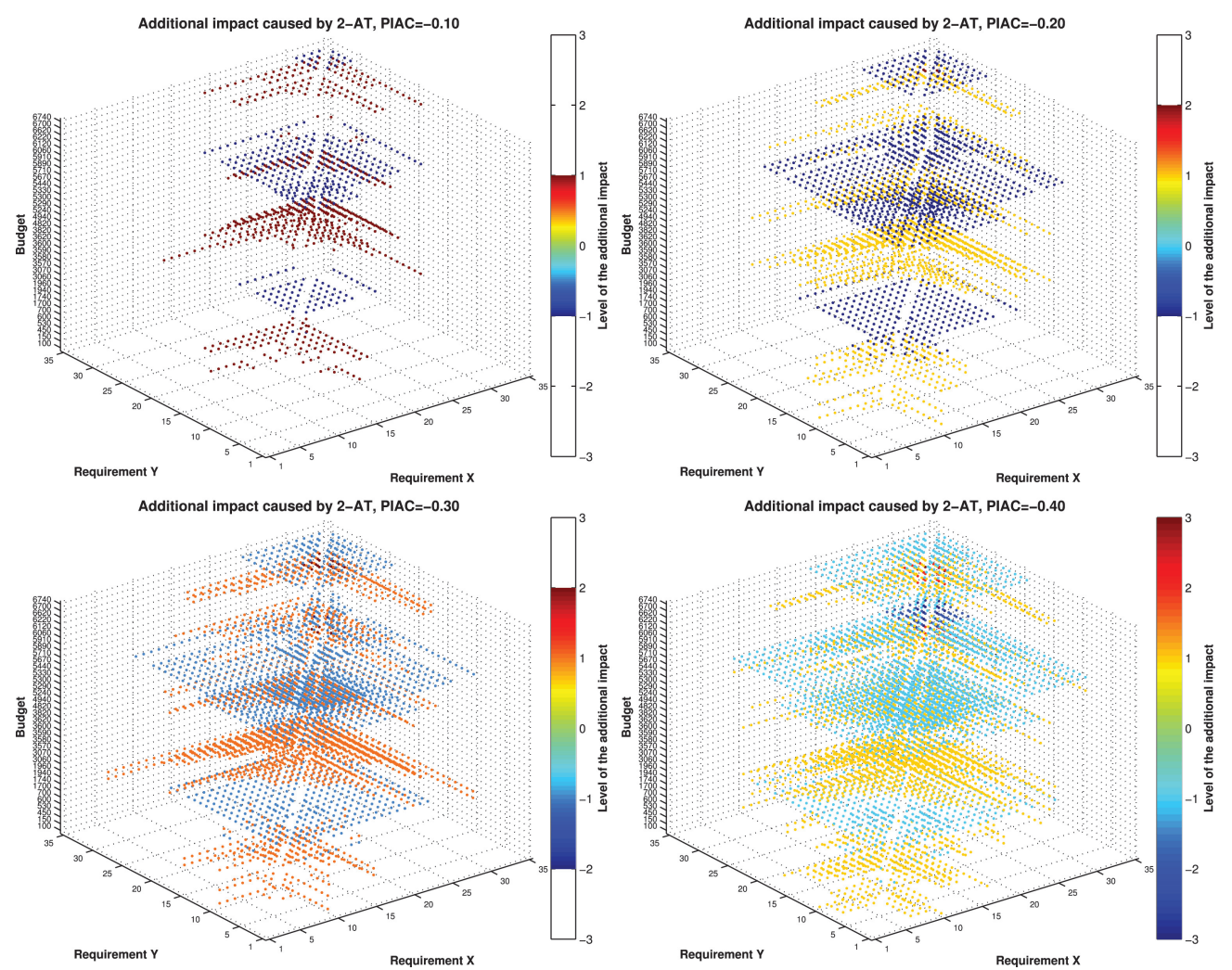

Fig. 13. Additional impact of second-order effects for negative PIAC (overestimated cost).

formulation used in the article has been widely studied [Bagnall et al. 2001; van den Akker et al. 2004, 2005; Zhang et al. 2008]: our experimentally results provide supporting evidence that there exists at least one exact algorithm, which is scalable, for a well-known formulation of NRP. The results for the Motorola dataset give an indication that the work can provide insights useful to a decision maker, but they constitute only existential evidence that this can occur in practice they do not guarantee that in all cases it will be possible to gain such insights. In this way, the results are case study based and we cannot generalize from them. It should also be noted that the Motorola dataset contains only a trivial set of dependencies; where there are more elaborate dependencies, these will also need to be taken into account, and this will affect the formulation of the problem.

Threats to construct validity concerns whether the measurement we made represent the actual problem. Real estimation errors in NRP can involve more than one requirement at a time, which would require more complicated modeling of the issue. However, we base our experiment on a widely studied and accepted sensitivity analysis technique (one-at-a-time) [Saltelli et al. 2000]. In any case, an exhaustive sensitivity analysis where an arbitrary number of requirements can be perturbed at the same time involves an exponential number of instances. Unfortunately, any method reducing this number could leave aside a sensitive instance.

We used 15 different problem sizes ranging from 100 to 1500 requirements with steps of 100 and correlation factors ranging from $0 \%$ to $100 \%$ in $5 \%$ steps. This resulted in 315 problem configurations, but we cannot claim that these are necessarily representative. 
Of course, further experimentation with alternative settings is always possible, and we cannot rule out the possibility that such experiments might yield different results. Though we can be more sure about performance between the values of the settings we chose, we can say nothing about scalability beyond 1,500 requirements. In many cases, such a large number of requirements would be sufficient, but should applications develop that required scalability beyond these numbers, then further experimentation would be required.

\section{RELATED WORK}

Bagnall et al. [2001] coined the term 'Next Release Problem (NRP)', formulating the consideration of the requirements for a software release as a search-based selection problem. However, there had been previous work on the application of optimization techniques for requirements prioritization [Karlsson et al. 1998]. The problem is also known as Software Release Planning [Ruhe and Greer 2003; Greer and Ruhe 2004]. There has been a recent overview of the area [Zhang et al. 2008] as well as a detailed survey of Search-Based Software Engineering (SBSE) techniques, which includes a section on the NRP [Harman et al. 2012b]. The work by Bagnall et al. [2001] is one of the few papers to consider an exact algorithm for the NRP. They use a standard integer programming formulation and present results from its implementation in the popular tool CPLEX [Bixby et al. 2000]. However, they do not present any results regarding the scalability of their approach.

While CPLEX is a widely used and robust tool, van den Akker et al. [2004, 2005] add various 'managerial steering inputs' to a basic ILP formulation of the NRP to provide greater flexibility. Li et al. [2007] present two integer linear programming models. The first of these is concerned with project management, seeking to reduce project completion time. This is a separate problem from the requirements analysis problem and one that has been widely studied elsewhere in the literature on SBSE for project management [Alba and Chicano 2007; Alvarez-Valdés et al. 2006; Antoniol et al. 2004, 2005; Chao et al. 1993; Kapur et al. 2008]. The second model integrates 'on time delivery' with maximal revenue generation. The article reports the results of experiments with both approaches on synthetic data.

No exact algorithm has been used in the literature on the NRP for which real-world problems were used in its evaluation. Indeed, most of the previous work on both real and synthetic data has concerned meta-heuristic algorithms which, though flexible and popular, are inexact and therefore cannot be used for a fully reliable sensitivity analysis.

Our approach to RSA uses Nemhauser-Ullmann's (NU) algorithm to solve multiple instances of the NRP. NU can be regarded as a smart rendition of dynamic programming by costs. This algorithm first appeared in the solution of capital allocation problems [Nemhauser and Ullmann 1969]. Many researchers had noticed that the algorithm seemed to behave well in practice when solving random KP instances, but a theoretical justification of these observations was lacking until groundbreaking work by Beier and Vöcking [2004] provided an explanation of the behavior of this algorithm in the average case under quite general conditions.

Karlsson et al. [1998] uses the Analytical Hierarchy Process (AHP) which allows for human contributions to the choice of ranking. This approach has been implemented in the Focal Point requirements analysis tool, which is now marketed by Telelogic, a subsidiary of IBM. Feather and Menzies [2002] used simulated annealing to solve requirements selection and optimization for a NASA project. Ruhe et al. [Greer and Ruhe 2004; Ruhe and Greer 2003; Ruhe and Ngo-The 2004] used a genetic algorithm to select requirements and represented results of the application of this approach to a real-world dataset. Ngo-The and Ruhe [2009] combine integer linear programming 
with a genetic algorithm to overcome the weaknesses of genetic algorithms with a twophase approach. Baker et al. [2006] also consider requirements problems as a selection problem, presenting results for simulated annealing and greedy algorithms on the Motorola dataset used in the present paper. AlBourae et al. [2006] also use both greedy and AHP algorithms in a release re-planning approach. Jalali et al. [2008] use a greedy algorithm to address the problem of risk reduction, where risks are characterized in terms of the risk of introducing new requirements.

Thus, previous work demonstrates the value of applying optimization techniques to the problem of requirements analysis. Recent work has shown that the results obtained by these approaches are superior to those assessments that can be made by a human in their optimization of choices [de Souza et al. 2010]. These "human-competitive" findings provide further evidence for the importance of optimization in requirements analysis.

However, when it comes to the problem of sensitivity, the inherent stochastic nature of all of the algorithms used means that they are inherently unreliable; we shall not know whether fluctuations are due to sensitivity or to the algorithms natural stochastic properties. The primary difference in the approach adopted in the present article lies in its use of a scalable exact algorithm for the NRP and its use as a "precise instrument" with which to address the sensitivity of a solution to the potential imprecision in the estimates upon which it is based.

This article concerns a single objective formulation of the NRP in which the problem is to find a set of requirements that maximize revenue while falling within budget. However, the underlying algorithm used for exact NRP solutions could be used to solve the bi-objective NRP problem. This may lead to extensions of the work in this article to consider multi-objective formulations of the NRP.

Zhang et al. [2007] introduced a multi-objective formulation in terms of a cost-benefit trade-off. In this approach the budget is not fixed. Instead, budget minimization becomes an additional objective. Saliu and Ruhe [2007a] also introduced a multi-objective formulation in which the balance was between concerns at two levels of abstraction: implementation and requirement, rather than between cost and value. Finkelstein et al. [2008a] used a multiple objective approach to analyze fairness in requirements. In this approach, the objective is to minimize cost while simultaneously maximizing fairness according to several different formulations of fairness; each of the different notions of fairness corresponds to an objective. Feather et al. [2004, 2006] also used a form of multi-objective visualization of the results from their simulated annealing approach, in which results are presented on a Pareto front.

In this article, we use a sensitivity analysis approach. Sensitivity analysis (SA) is found in other areas of engineer but is not widely used, hitherto, in requirements engineering. Other applications of SA are widely found in the literature for various areas, such as chemical kinetics [Sandu et al. 2003], physical science [Newman et al. 1999], environmental modeling [Hamby 1994], telecommunications engineering [Racu et al. 2005], and financial analysis [Levine and Renelt 1992]. In software engineering, the application of sensitivity analysis has been mostly focused on the area of software reliability and prediction models [Rodrigues et al. 2005; Wagner 2007a, 2007b; Zhu et al. 2005]. Harman et al. presented a search-based sensitivity analysis of NRP [2009]. However, precise sensitivity analysis requires exact algorithms in order to avoid unwanted and potentially ruinous 'noise' from the approximate nature of the algorithm.

\section{IMPLICATIONS OF THE RESULTS FOR WORK ON NRP}

The results in this article have implications for subsequent work on the NRP and release planning. Most previous work has been concerned with giving insight to decision makers about possible choices of requirement. For these applications, the inherent imprecision of metaheuristic methods may not be an issue. 
In some circumstances, such as the exploration of trade-offs between different objectives in multiobjective formulations [Finkelstein et al. 2008b; Saliu and Ruhe 2007b], it may be sufficient to use inexact algorithms, which may offer other benefits (such as handling messy, incomplete data) or where these formations have no known precise solution approach that can be computed in reasonable time.

However, for the application of optimization-based approaches to problems concerning the RSA, it is important to have an algorithm that guarantees globally optimal solutions at the heart of the approach; to assess the impact of estimate inaccuracies, we need an accurate approach. Without an optimal algorithm, it will not be clear whether sensitivity observed in solutions obtained is due to PIACs or whether it arises due to the inherent stochastic nature of the algorithm used. In particular, that the previous work on sensitivity analysis using a genetic algorithm [Harman et al. 2009], suffers from this problem.

This does not mean that metaheuristic approach cannot be used for any aspect of RSA. As we discuss in the future work for this research agenda, for higher-order effects (interactions between estimate inaccuracies), it may prove essential to use a metaheuristic approaches to cater for the scale of the space of possible interactions. However, in such a scenario, it may be unrealistic to expect that a complete characterization of all estimate inaccuracy risks can be captured.

\section{FUTURE WORK}

Future work will consider different formulations of the requirements problem, including those with complex dependencies between requirements, for example, where the value and cost of one requirement are affected by the other requirements also included in the release of the software.

Handling higher-order effects for orders $n,(n>2)$ remains an interesting open problem for future work. In this article, we showed how a one-at-a-time analysis (OAT) could be used to handle all possible single estimate inaccuracies and how this could be extended to all second-order interaction effects between estimate inaccuracies. Approaches to higher orders of interactions require very different approaches, since they will not be so easy to visualize and the computational complexity grows exponentially with the interaction order. One interesting avenue for future work will consist of using SBSE to search the space of higher-order interactions to locate potential problematic cases.

Other requirements selection algorithms, such as OPTIMIZE RASORP $_{\text {[Ngo-The and }}$ Ruhe 2009], could also be considered in future work to determine whether or not they could form a suitably precise foundation on which to build sensitivity analyses.

\section{CONCLUSIONS}

There has been a lot of recent interest in the application of search-based software engineering (SBSE) to requirements analysis optimization. One important goal of this work has been to find algorithms that are able to select an ideal set of requirements for the next release of the system, an activity known as release planning for an optimization problem known as the Next Release Problem (NRP). Recent work has demonstrated human competitive results for this area of SBSE. However, there remains a problem: the optimization can only ever be as good as the quality of the estimates upon which it is based. Software engineering estimation inaccuracy is widely believed to be significant, making this an important problem.

In this article, we introduce a one-at-a-time (OAT) sensitivity analysis, incorporating a scalable exact optimization algorithm as the NRP Solver at the heart of the analysis. We demonstrated that this exact algorithm can be used to precisely assess the sensitivity of an instance of the NRP to inaccuracies in its estimate. This allows the 
requirements engineer to locate relatively risk-free 'insensitive' budget choices and to identify those 'sensitive' requirements for which estimates are particularly important. Scalability is clearly important, but it is difficult, because the NRP is NP-hard. We presented results from an experimental study that demonstrate the scalability of our approach, together with a real-world case study that illustrates the way in which our approach can assist a requirements engineer. We also illustrate that analysis of higher-order estimate inaccuracy is feasible using an exact algorithm.

Armed with a reliable assessment of sensitivity, the requirements analyst can better account for the impact of estimate inaccuracies, thereby making better informed choices in the crucial early stages of the software development process. The case study scenarios in this article show how our analysis can reveal particularly sensitive budget levels and requirements that might otherwise have gone unnoticed by the requirements decision maker.

\section{ACKNOWLEDGMENTS}

We are grateful to the referees for suggesting many ways of improving the original manuscript. In particular, one of the referees drew our attention to the importance of considering higher-order effects, which occasioned a major revision to this article.

\section{REFERENCES}

E. Alba and F. Chicano. 2007. Software project management with GAs. Inf. Sci. 177, 11, 2380-2401.

T. Albourae, G. Ruhe, and M. Moussavi. 2006. Lightweight replanning of software product releases. In Proceedings of the 1st International Workshop on Software Product Management (IWSPM'06). IEEE Computer Society, 27-34.

R. Alvarez-Valdés, E. Crespo, J. M. Tamarit, and F. Villa. 2006. A scatter search algorithm for project scheduling under partially renewable resources. J. Heuristics 12, 1-2, 95-113.

G. Antoniol, M. Di Penta, and M. Harman. 2004. A robust search-based approach to project management in the presence of abandonment, rework, error and uncertainty. In Proceedings of the 10th International Symposium on the Software Metrics (METRICS'04). IEEE Computer Society, 172-183.

G. Antoniol, M. D. Penta, and M. Harman. 2005. Search-based techniques applied to optimization of project planning for a massive maintenance project. In Proceedings of the 21st IEEE International Conference on Software Maintenance (ICSM'05). IEEE Computer Society, Los Alamitos, CA, 240-249.

A. Bagnall, V. Rayward-Smith, and I. Whittley. 2001. The next release problem. Inf. Softw. Technol. 43, 14, 883-890.

P. Baker, M. Harman, K. Steinhofel, and A. Skaliotis. 2006. Search based approaches to component selection and prioritization for the next release problem. In Proceedings of the 22nd IEEE International Conference on Software Maintenance (ICSM'06). IEEE Computer Society, 176-185.

R. Beier and B. Vöcking. 2004. Random knapsack in expected polynomial time. J. Comput. Syst. Sci. 69, 3, 306-329.

R. E. Bixby, M. Fenelon, and Z. Gu. 2000. MIP: Theory and practice-closing the gap. Syst. Model. Optim.: Methods, Theory, Appl. 1, 1960, 1-31.

C. Chao, J. Komada, Q. Liu, M. Muteja, Y. Alsalqan, and C. Chang 1993. An application of genetic algorithms to software project management. In Proceedings of the 9th International Advanced Science and Technology. 247-252.

B. H. Cheng and J. M. Atlee. 2007. Research directions in requirements engineering. In Proceedings of the Future of Software Engineering. IEEE Computer Society, Los Alamitos, CA, 285-303.

T. E. Colanzi, S. R. Vergilio, W. K. G. Assuno, and A. Pozo. 2012. Search based software engineering: Review and analysis of the field in Brazil. J. Syst. Softw.

J. T. de Souza, C. L. Maia, F. G. de Freitas, and D. P. Coutinho. 2010. The human competitiveness of search based software engineering. In Proceedings of the 2nd International Symposium on Search Based Software Engineering (SSBSE'10). IEEE Computer Society.

M. S. Feather, S. L. Cornford, J. D. Kiper, and T. Menzies. 2006. Experiences using visualization techniques to present requirements, risks to them, and options for risk mitigation. In Proceedings of the International Workshop on Requirements Engineering Visualization (REV'06). IEEE, 10-10. 
M. S. Feather, J. D. Kiper, and S. Kalafat. 2004. Combining heuristic search, visualization and data mining for exploration of system design space. In Proceedings of the 14th Annual International Symposium of International Council on Systems Engineering (INCOSE'04).

M. S. Feather and T. Menzies. 2002. Converging on the optimal attainment of requirements. In Proceedings of the 10th IEEE International Conference on Requirements Engineering (RE'02). IEEE, 263-270.

A. Finkelstein, M. Harman, S. A. Mansouri, J. Ren, and Y. Zhang. 2008a. Fairness analysis in requirements assignments. In Proceedings of the 16th IEEE International Requirements Engineering Conference (RE'08). IEEE Computer Society, 115-124.

A. Finkelstein, M. Harman, S. A. Mansouri, J. Ren, and Y. Zhang. 2008b. Fairness analysis in requirements assignments. In Proceedings of the 16th IEEE International Requirements Engineering Conference (RE'08).

F. G. Freitas and J. T. Souza. 2011. Ten years of search based software engineering: A bibliometric analysis. In Proceedings of the 3rd International Symposium on Search Based Software Engineering (SSBSE'11). 18-32.

H. C. Frey and S. Patil. 2002. Identification and review of sensitivity analysis methods. Risk Anal. 22, 3, $553-578$.

M. R. Garey and D. S. Johnson. 1978. Strong NP-completeness results: Motivation, examples, and implications. J. ACM 25, 499-508.

M. R. Garey and D. S. Johnson. 1979. Computers and Intractability: A Guide to the Theory of NPCompleteness. W. H. Freeman.

D. Greer and G. Ruhe. 2004. Software release planning: An evolutionary and iterative approach. Inf. Softw. Technol. 46, 4, 243-253.

D. M. Hamby. 1994. A review of techniques for parameter sensitivity analysis of environmental models. Environ. Monitor. Assess. 32, 2, 135-154.

M. Harman. 2007. The current state and future of search based software engineering. In Proceedings of the Future of Software Engineering, L. Briand and A. Wolf, Eds. IEEE Computer Society Press, Los Alamitos, CA, 342-357.

M. Harman, E. Burke, J. A. Clark, and X. Yao. 2012a. Dynamic adaptive search based software engineering. In Proceedings of the 6th IEEE International Symposium on Empirical Software Engineering and Measurement (ESEM'12).

M. Harman, J. Krinke, J. Ren, and S. Yoo. 2009. Search based data sensitivity analysis applied to requirement engineering. In Proceedings of the 11th Annual Conference on Genetic and Evolutionary Computation (GECCO'09). ACM, 1681-1688.

M. Harman, A. Mansouri, and Y. Zhang. 2012b. Search based software engineering: Trends, techniques and applications. ACM Comput. Surv. 45, 1, Article 11.

M. Harman, P. Mcminn, J. Souza, and S. Yoo. 2012c. Search based software engineering: Techniques, taxonomy, tutorial. In Empirical Software Engineering and Verification: (LASER'09-10), B. Meyer and M. Nordio, Eds., Lecture Notes in Computer Science, vol. 7007, Springer, 1-59.

J. Helton, J. Johnson, C. Sallaberry, and C. Storlie. 2006. Survey of sampling-based methods for uncertainty and sensitivity analysis. Reliability Eng. Syst. Safety 91, 10-11, 1175-1209.

O. Jalali, T. Menzies, and M. Feather. 2008. Optimizing requirements decisions with KEYS. In Proceedings of the 4th International Workshop on Predictor Models in Software Engineering (PROMISE'08). ACM, 79-86.

P. Kapur, A. Ngo-The, G. Ruhe, and A. Smith. 2008. Optimized staffing for product releases and its application at Chartwell Technology. J. Softw. Maintenance Evol. Res. Pract. (Special Issue Search Based Software Engineering), 20, 5, 365-386.

J. Karlsson, C. Wohlin, and B. Regnell. 1998. An evaluation of methods for priorizing software requirements. Info. Softw. Technol. 39, 14-15, 939-947.

R. Karp. 1972. Reducibility among combinatorial problems. Complex. Comput. Compu. 40, 4, 85-103.

R. Levine and D. Renelt. 1992. A sensitivity analysis of cross-country growth regressions. Amer. Econ. Rev. 82, 4, 942-963.

C. Li, M. van den Akker, S. Brinkkemper, and G. Diepen. 2007. Integrated requirement selection and scheduling for the release planning of a software product. In Proceedings of the 13th International Working Conference on Requirements Engineering: Foundation for Software Quality (RefsQ'07). Lecture Notes in Computer Science, vol. 4542. Springer, 93-108.

G. L. Nemhauser and Z. Ullmann. 1969. Discrete dynamic programming and capital allocation. Manage. Sci. 15, 9, 494-505. 
J. Newman, A. Taylor, R. Barnwell, and P. Newman. 1999. Overview of sensitivity analysis and shape optimization for complex aerodynamic configurations. J. Aircraft 36, 1, 87-96.

A. Ngo-The and G. Ruhe. 2009. Optimized resource allocation for software release planning. IEEE Trans. Softw. Eng. 35, 1, 109-123.

D. Pisinger. 2005. Where are the hard knapsack problems? Comput. Oper. Res. 32, 9, 2271-2284.

R. Racu, M. Jersak, and R. Ernst. 2005. Applying sensitivity analysis in real-time distributed systems. In Proceedings of the 11th IEEE Real Time and Embedded Technology and Applications Symposium. IEEE Computer Society, 160-169.

G. N. Rodrigues, D. S. Rosenblum, and S. Uchitel. 2005. Sensitivity analysis for a scenario-based reliability prediction model. In Proceedings of the Workshop on Architecting Dependable Systems (WADS'05). ACM, New York, NY, 1-5.

G. Ruhe, A. Eberlein, and D. Pfahl. 2003. Trade-off analysis for requirements selection. Int. J. Softw. Eng. Knowl. Eng. 13, 4, 345-366.

G. Ruhe and D. Greer. 2003. Quantitative studies in software release planning under risk and resource constraints. In Proceedings of the International Symposium on Empirical Software Engineering (ISESE'03). IEEE, 262-270.

G. Ruhe and A. Ngo-The. 2004. Hybrid intelligence in software release planning. Int. J. Hybrid Intell. Syst. 1, 1-2, 99-110.

M. O. Saliu and G. Ruhe. 2007a. Bi-objective release planning for evolving software systems. In Proceedings of the the 6th Joint Meeting of the European Software Engineering Conference and the ACM SIGSOFT Symposium on the Foundations of Software Engineering (ESEC-FSE'07). ACM, New York, NY, 105-114.

M. O. Saliu and G. Ruhe. 2007b. Bi-objective release planning for evolving software systems. In Proceedings of the 6th Joint Meeting of the European Software Engineering Conference and the ACM SIGSOFT International Symposium on Foundations of Software Engineering (ESEC/FSE'07). I. Crnkovic and A. Bertolino, Eds., ACM, 105-114.

A. Saltelli and P. Annoni. 2010. How to avoid a perfunctory sensitivity analysis. Environ. Model. Softw. 25, 12, 1508-1517.

A. Saltelli, M. Ratto, T. Andres, F. Campolongo, J. Cariboni, D. Gatelli, M. Saisana, and S. Tarantola. 2008. Global Sensitivity Analysis: The Primer. John Wiley \& Sons, Sussex.

A. Saltelli, S. Tarantola, and F. Campolongo. 2000. Sensitivity analysis as an ingredient of modeling. Stat. Sci. 15, 4, 377-395.

A. Sandu, D. N. Daescu, and G. R. Carmichael. 2003. Direct and adjoint sensitivity analysis of chemical kinetic systems with KPP: Part I-theory and software tools. Atmospheric Environ. 37, 36, 5083-5096.

M. Shepperd. 2007. Software project economics: A roadmap. In Proceedings of the Future of Software Engineering (FoSE'07). IEEE Computer Society, 304-315.

M. van den Akker, S. Brinkkemper, G. Diepen, and J. Versendaal. 2004. Flexible release composition using integer linear programming. Tech. rep. UU-CS-2004-063, Institute of Information and Computing Sciences, Utrecht University.

M. van den Akker, S. Brinkkemper, G. Diepen, and J. Versendaal. 2005. Flexible release planning using integer linear programming. In Proceedings of the 11th International Workshop on Requirements Engineering for Software Quality (RefsQ'05). Essener Informatik Beitrage, 247-262.

S. Wagner. 2007a. An approach to global sensitivity analysis: FAST on COCOMO. In Proceedings of the 1st International Symposium on Empirical Software Engineering and Measurement (ESEM'O7). IEEE Computer Society, Los Alamitos, CA, 440-442.

S. Wagner. 2007b. Global sensitivity analysis of predictor models in software engineering. In Proceedings of the 3rd International Workshop on Predictor Models in Software Engineering (PROMISE'O7). IEEE Computer Society, 3-10.

Y. Zhang, A. Finkelstein, and M. Harman. 2008. Search based requirements optimisation: Existing work and challenges. In Proceedings of the International Working Conference on Requirements Engineering: Foundation for Software Quality (REFSQ'08). Lecture Notes in Computer Science, vol. 5025, Springer, 88-94.

Y. Zhang, M. Harman, and S. A. Mansouri. 2007. The multi-objective next release problem. In Proceedings of the Genetic and Evolutionary Computation Conference. ACM Press, New York, NY, 1129-1136.

L. Zhu, A. Aurum, I. Gorton, and R. Jeffery. 2005. Tradeoff and sensitivity analysis in software architecture evaluation using analytic hierarchy process. Softw. Quality Control 13, 4, 357-375.

Received May 2012; revised April, August 2013; accepted October 2013 